\title{
A Beacon in the Galaxy: Updated Arecibo Message for Potential FAST and SETI Projects
}

\author{
Jonathan H. Jiang ${ }^{1}$, Hanjie $\mathrm{Li}^{2}$, Matthew Chong ${ }^{3}$, Qitian Jin ${ }^{4}$, Philip E. Rosen ${ }^{5}$, Xiaoming Jiang ${ }^{6}$, Kristen A. Fahy ${ }^{1}$, \\ Stuart Taylor ${ }^{7}$ Zhihui Kong ${ }^{8}$, Jamilah Hah ${ }^{9}$, Zonghong Zhu ${ }^{8}$ \\ 1. Jet Propulsion Laboratory, California Institute of Technology, Pasadena, CA 91108, USA \\ 2. Virginia Polytechnic Institute and State University, Blacksburg, VA 24061, USA \\ 3. University of Cambridge, Cambridge CB2 1TN, United Kingdom \\ 4. Hanze University of Applied Sciences, 9747 AS Groningen, Netherlands \\ 5. Retired, Chevron Energy Technology Company, Houston, TX 77002, USA \\ 6. School of Physics and Technology, Wuhan University, Wuhan, Hubei, China 430072 \\ 7. SETI Institute, Mountain View, CA 94043, USA \\ 8. Department of Astronomy, Beijing Normal University, Beijing, China 100875 \\ 9. University of Southern California, Los Angeles, CA 90089, USA \\ Correspondence: Jonathan.H.Jiang@jpl.nasa.gov \\ Copyright@2022, California Institute of Technology, All Rights Reserved \\ Keywords: Galaxy, Interstellar, Radio Message, Civilization, Earth, Binary, Radio Telescope
}

\begin{abstract}
An updated, binary-coded message has been developed for transmission to extraterrestrial intelligences in the Milky Way galaxy. The proposed message includes basic mathematical and physical concepts to establish a universal means of communication followed by information on the biochemical composition of life on Earth, the Solar System's time-stamped position in the Milky Way relative to known globular clusters, as well as digitized depictions of the Solar System, and Earth's surface. The message concludes with digitized images of the human form, along with an invitation for any receiving intelligences to respond. Calculation of the optimal timing during a given calendar year is specified for potential future transmission from both the Five-hundred-meter Aperture Spherical radio Telescope in China and the SETI Institute's Allen Telescope Array in northern California to a selected region of the Milky Way which has been proposed as the most likely for life to have developed. These powerful new beacons, the successors to the Arecibo radio telescope which transmitted the 1974 message upon which this expanded communication is in part based, can carry forward Arecibo's legacy into the $21^{\text {st }}$ century with this equally well-constructed communication from Earth's technological civilization.
\end{abstract}

\section{Introduction}

Since the first faint flickering of sentience dawned in the primal minds of modern humans' distant ancestors some hundred thousand generations ago, we have sought to communicate. Cooperation facilitated by primitive grunts and gestures may well have been the difference between extinction on the East African veldt and eventual mastery of the Earth. As survival gave way to dominance, attained at such cost, humanity's path toward civilization lay open. With the watershed inventions of written language, mathematics, and the scientific method, generational construction of complex ideas, concepts and innovations became possible. Driven by broader inquiry, ancient scholars gazed at the stars wheeling through the vault of night and inevitably confronted what is perhaps the most profound of all questions: Are we alone or are those points of light in the sky home to others we may yet come to know? It would take five millennium to progress from Sumerian cuneiform to the great radio telescopes of the $20^{\text {th }}$ and $21^{\text {st }}$ centuries - and with that, the means to finally begin seeking out an answer. 
Even before the first exoplanet discovery was confirmed in 1995, attempts at listening for signals of extraterrestrial intelligent (ETI) origin, as well as sending signals of our own, were well underway. Despite a few false alarms such as the first detection of what turned out to be pulsars in the 1960s and the "WOW Signal" in 1977, we have listened with increasingly sophisticated technology for any utterance from a far-flung 'other'. As well, we have sent signals, both by radio and by the far slower physical couriers Pioneer and Voyager to any beings who may share the Milky Way galaxy with us. Standing out among these first bold attempts, though, is the Arecibo Message, transmitted in 1974 as a beamed radio signal at wavelength 126 millimeters towards the M13 globular cluster some 25,000 lightyears distant. Constrained by the universal speed limit of light in vacuum, the electromagnetic waves conveying the Arecibo Message have traversed less than $0.2 \%$ of the distance to their intended target. While the nearly inconceivable vastness of interstellar space may be humbling, rather than deter humanity from pressing ever forward with our quest to communicate beyond our home world it should be taken to heart as a challenge. As Carl Sagan so eloquently stated, we are indeed "star stuff contemplating the stars." Communicating with other civilizations is the logical goal of Sagan's proclamation. The skies above us today, not so unlike the world which lay just over the East African horizon two million years ago, invite our best efforts to pursue with renewed conviction and better means those answers we instinctually seek.

In southwest China the Five-hundred-meter Aperture Spherical radio Telescope ("FAST"), otherwise known as Tianyan, is one successor to the recently decommissioned and disassembled Arecibo radio telescope. The illuminated aperture of the FAST is $300 \mathrm{~m}$, and its overall performance and sensitivity are several times higher than was Arecibo's and those of the other existing radio telescopes [RRA 2020]. The FAST comprises 2,225 actuators and cable net, which forms a complex coupling active reflector system. A 30-ton feed cabin, which is driven by six cables, is positioned about $140 \mathrm{~m}$ above the reflector. Another, the SETI Institute's Allen Telescope Array ("ATA") in northern California, is operational as well and will eventually come to include as many as 350 individual dish antennae operating in concert to both receive and send signals using cutting edge technology. It is understood that both FAST and SETI's ATA are currently receive-only radio telescopes. Both may possibility be upgraded through future enhancements that will enable the transmission of messages as well. If so profound a goal as communication with alien civilizations is to be realized the powerful tools of FAST and ATA must be paired with an equally well designed and constructed message to transmit. How intelligences not of this Earth would decode and interpret our message, this contained within the constraints of data compression and the logic-demanding simplicity of binary coding, is key to crafting a worthy successor to the Arecibo Message of nearly half a century ago. With these considerations firmly in mind, we present the structure and content of a message to send from humanity's beacon in the galaxy.

\section{Methodology}

In the last nearly 50 years, multiple communiques to ETI have been developed and broadcast into space, each more advanced than the last. The first message, as contained in the Arecibo Transmission, became a template for future messages. Sent entirely in binary, the 1974 Arecibo Message portrays our base-10 mathematics system, the most common elements to humans, and our Solar System - including the location of our life-bearing Earth. In the few years before and after the Arecibo Message, the Pioneer and Voyager spacecraft were launched containing plaques and discs that innovated the idea of using the $\mathrm{H}_{2}$ molecule spin-flip transition as the metric for length and time; they also introduced the idea of sending cultural information - such as human 
greetings - to ETI. Of the more recent, the Evpatoria Transmission Messages (ETMs,) sent in 1999 and 2003, also transmitted in binary, invented an easily distinguishable alphabet system and included an exhaustive list of our basic mathematics and physics knowledge. Most importantly, the ETMs included an invitation to reply to the message with questions directed to the receiving ETI.

In this section we will detail the development of an updated and modernized Arecibo Message, including selection criteria used in choosing the types of information in the message and the rationale for each, citing the imperative to consider the reverse circumstance as a kind of logic test - i.e., what would humans want a message from an ETI to contain?

\subsection{The Message Contents}

On the golden phonograph record attached to each of the two Voyager spacecraft launched in 1977, greetings in 55 languages were recorded, as were photographs of the Earth and of human culture. The Beacon in the Galaxy (BITG) message will be sent as a beamed radio wave coded in binary; recognizing the shortcomings of such means no similar voice or language recordings will be included in the BITG message. However, the message could feasibly contain coded depictions of great cultural works of art and architecture and/or images of nature such as forests, mountains, and oceans. The limitations on including such is due to the constraints on the size of the message as well as the importance we anticipate ETI would place on these images. Each image would require at least a $128 \times 128$ bits resolution which would greatly increase the transmission length of the message - not only increasing the energy required to beam the message but also greatly increasing the probability of error during the transmission, travel, and receiving of the message. Finally, if humanity were receiving a message containing these depictions it is not clear we would understand what they meant. Thus, the decision has been made to exclude all mentions of human culture and language, instead focusing on concepts any ETI capable of receiving and decoding the message would necessarily understand: mathematics and physics.

Though the concept of mathematics in human terms is potentially unrecognizable to ETI, binary is likely universal across all intelligence. Binary is the simplest form of mathematics as it involves only two opposing states: zero and one, yes or no, black or white, mass or empty space. Hence, the transmission of the code as binary would very likely be understandable to all ETI and is the basis of the BITG message. With binary, the two best candidates for headers would be a series of " 1 "s followed by a series of " 0 "s or a series of prime number composed of alternating “0”s and "1"s - i.e., 00111000001111111 . Prime numbers can be safely assumed to be unique to intelligent construction given there being no known naturally occurring phenomena in the cosmos which generates that particular series and would thus signal any ETI that something of intelligent origin is contained within this signal. However, if used as the header line this could cause confusion during decoding and also has a higher likelihood of error during the transmission or travel of the message. It would also result in a header line being a different length than the rest of the message lines, incurring increased difficulty decoding and potentially lowered intuition of the message for ETI. As such, the header will consist of $\mathrm{x} 0 \mathrm{~s}$ followed by $\mathrm{x} 1 \mathrm{~s}$ then $\mathrm{x} 0 \mathrm{~s}$ again such as: 000000111111000000 , with the start of each indicating the length of each line of the message.

Following the header, and with the idea of binary being universal in mind, is the depiction of homo sapiens' base-10 mathematics system. Explanation of the foundations of our mathematics system provide the basis for creating basic measurement units understandable to ETI and are critical to communicating further types of information. By describing our mathematics basis in terms of binary, ETIs would be able to follow along with the mathematics described in the rest of 
the message. Following this concept, the message would need to contain mathematical operators such as the addition, subtraction, division, and multiplication symbols. However, as in the Evpatoria Transmissions, we found that using the conventional symbols for many mathematical operators is highly prone to interpretation and transmission error. Thus, the BITG message will include identical formatting in the "alphabet" from the Evpatoria Transmissions for concepts including: the aforementioned operators, equals sign, dot symbol, meters, elements, and seconds. Other aspects of the Evpatoria Transmission Message Alphabet (ETMA) such as $\pi$, radius and other geometric concepts are omitted from the BITG message as geometry is simply a logical progression from more basic concepts of mathematics and needn't lengthen the message with content ETI would likely already understand.

The transmission of units and basic data is one of the most important parts of the message to consider and plan carefully. It is highly improbable that any ETI would use similar measurements to the metric system - not only can humans not decide on any singular measuring system, but it is probable that ETI do not use our base-10 mathematics. Hence, the depiction of our units must be done through universal natural constants: the Hydrogen spectrum, the $\mathrm{H}_{2}$ molecule, and the Helium atom. For any life to exist, a host star must exist to create energy and hence the physical characteristics of Hydrogen and Helium would surely be known to ETI. Additionally, the Hydrogen spectrum is one of the most important discoveries of physical chemistry, and very distinct - it would be hard to mistake the spectrum lines of the most abundant element in the universe for anything else. Finally, the $\mathrm{H}_{2}$ molecule's spin-flip transition is constant, easily recognizable and can be used to describe both time and length as was done on the Voyager and Pioneer Plaques. These three basic metrics allow the message to include common elements, DNA/double helix, basic physical concepts, data in familiar (e.g., metric) units, and a timestamp for the transmission of the BITG message.

The inclusion of a timestamp is imperative in detailing to any ETI such that if/when they send a return message they can know, when coupled with Earth's present location in the Milky Way, where to beam their reply and when to expect their message to arrive back to humanity. The timestamp can be done by quantifying the age of the universe down to the year. Most importantly, a timeline originating from the Big Bang would be crucial for indicating the universal time our signal was sent. While dating to the decade would be more data economical, the extra detail provided from counting to the closest year is worth the extra transmission length. Anything more or less accurate would either be overly specific and redundant or potentially too easily confused with other message components. Intertwined with the timestamp is the idea of including a human civilization timeline, consisting of key moments in history such as the birth of civilization or transformative discoveries and inventions. Such a timeline would thus contain both important scientific dates as well as points that advanced humanity - e.g., Newton discovering his Laws of motion and force, Einstein's Relativity, the start of the Space Age and human moon landing. Every single achievement included in the timeline would have to be depicted by an image, approximately $128 \times 128$ bits at a minimum, or the resolution would likely be too indistinct. Given how many important historical milestones exist, the message would simply become too long to make this a viable option.

Just as the header doubles as a "notice me" signal and a line length indicator, the timestamp doubles as the beginning of the more advanced content of the message. Between the header and timestamp, elementary concepts meant for conveying basic physical ideas establish a basis for communication. The content after the timestamp goes into far more depth and requires a combination of the information contained earlier in the message. The first such section builds off 
the idea of the Hydrogen spectrum and Helium atom by explaining our most critical chemical elements for terrestrial life. By representing Hydrogen and Helium as the numbers 1 U0 (1 proton, 0 neutron) and 2U2 (2 protons, 2 neutrons), respectively, we can describe the other common elements using their respective atomic numbers and most commonly occurring isotopes. Further development of the concept of mass can be done by way of the mass of protons and neutrons. While this section is modeled off the ETM, we have chosen to leave off the concept of Avogadro's Number as it would likely be unnecessarily confusing for the ETI receiver to grasp absent more chemistry context. This idea of elements is included to describe what our most common constituent atoms are and to lay the groundwork for describing human biology - without first introducing elements it would not be possible to describe the chemical makeup of humans, including DNA and its distinct double-helix structure.

Similar to the ETM, the BITG message includes a visual depiction of the four constituent bases of DNA: adenosine, cytidine, guanosine, and thymidine. Additionally, the concept of amino acids and the formula of glucose is included to give a more holistic view of the most important biochemistry in humans and life on Earth in general. Immediately following is a double-helix picture and that of the double-helix within a cell. This provides the information humans, among other life on Earth, are a carbon-based form, this to better start a conversation, and additionally to infer how life on Earth came to be via complex self-replicating organic molecules. The logical follow up to the basal makeup of humans is a depiction of our physical form, along with basic data like height and our population at time of transmission. Exclusion of details such as auditory, temperature, and visual ranges is due to constraints on length and previous information - the message does not include an explanation of our units of hearing frequency $(\mathrm{Hz})$ or temperature (degrees) as our basis and units will likely be much different from any ETI. This information is part of the basic expectation of any message to an ETI as it allows recognition of our appearance - a detail of a given ETI that would no doubt be of interest to us, and relevant should further exchanges be established, or we someday physically meet. Building off this idea, the BITG message contains a map of the Milky Way with directions to our Solar System for a sufficiently advanced ETI to discover us. Logically, we also include a map of the Solar System itself with indication of where the Earth is located relative to our host star and the other planets - along with a digitized map of Earth with relative portion of land to water. This provides the ETI with the necessary information to make contact with us either by sending a follow up transmission to Earth or, if their technology is sufficiently advanced, sending a physical vehicle to Earth at some point in the future.

The idea of a response from ETI is a prospect encouraged by a depiction of the transmitting telescope and another generic telescope design sending electromagnetic wave transmissions to each other. This encouragement for a response opens up future possibilities of messages to the same regions or the confirmation of ETIs within the rest of the Milky Way - both distinctively exciting prospects. An invitation for conversation is an ideal conclusion to any initial message exactly what humanity would want at the end of a message to us - and is followed by insertion of the header again, though this time as a footer. The header/footer pattern signifies an intelligenceorganized electromagnetic wave possessing a beginning, an end, and what is to be decoded as the message positioned within.

\subsubsection{Location Stamp to Indicate the Position of the Message Sender}

Before locating ourselves in interstellar space, it is necessary to establish a reference frame. In a satellite positioning system, the reference frame is from the satellites themselves. In principle, if we know the distance between one satellite and us, we can constrain our position on a sphere. If 
we then get two distances, we know our position on two spheres' intersection - a ring, and once we have three, we can locate ourselves within an intersection of three spheres - two points. From there, given some reasonable speculation, we can confirm at which of those two points we reside. The implication is we need a minimum of three reference stars, and if we have more stars our accuracy will be further enhanced.

To describe our location in the Milky Way a galactic scale reference frame is needed. However, given there are billions of stars in the galaxy this raises some questions to us - which stars should be selected? How best to identify them in the sky after long periods of stellar and galactic evolution as well as across large-scale travel? About half-century ago, Pioneer and Voyager's plaques used 14 pulsars to construct a reference frame, this owing to pulsars' periods of rotation being extremely stable - i.e., once we measure a given pulsar's period precisely, it can be used as that star's unique identifier. Unfortunately, this method has some intrinsic defects. Pulsar radiation is constrained as a beam rather than radiating uniformly in all directions, with the opening angle limiting the visible zone to a thin traced-out ring. After large-scale travel through time and space, if we leave the visible zone of a given pulsar, we will lose the pulsed signal and could even fail to notice a star is located there at all. Additionally, as Milky Way and its stars evolve, the position of stars, the galaxy's structure and the direction of pulsars' radiation beams will change. Thus, we should consider turning to a more isotropic and easily identifiable objects.

For the above application, globular clusters (GCs) could prove a superior choice per the following reasons:

a) GC radiation is isotropic, it can be seen from all directions in the galaxy except where obscured by interstellar dust and gas.

b) GCs are generally distributed in and around the central region of the galaxy; thus can be used both by local ETIs in our region of the Orion-Cygnus Arm and even those located on the far side of the Milky Way's central hub from Earth's perspective.

c) Hundreds of thousands of stars comprise a typical GC, thus the sum of their luminosity is much brighter than that of the typical pulsar. This property makes the GC a more observer friendly target, better supporting the intended large-scale construction of the reference frame.

d) GCs are multifaceted astronomical objects; their greater number of characterizing details helping to improve identification and thus serve as improved cosmic landmarks to pulsars.

As shown in the Figure 2.1, over 3,000 pulsars are known to be distributed in the Milky Way. Due to this large number of pulsars, it is easy to get confused about which small subset are referencing the target. When considering pulsar beams' limited opening angle, there must be many more pulsars that cannot be seen from Earth. Observational selection effects are also shown in the plot which illustrates how most known pulsars are near the Sun. This implies that there are far more undiscovered pulsars far from the Sun, especially behind the galaxy's central region. Determining which pulsars our message was referring to versus pulsars we had not discovered would inevitably prove confusing to distant ETIs performing reconstruction of navigation maps. In contrast, the globular clusters' distribution is more symmetric about the galactic center. The same globular clusters can be recognized over a much greater region of the galaxy, so their discoveries are applicable to more locations throughout the galaxy than pulsars. Thus, the distribution of globular clusters (GCD) can serve as a more general and more reliable reference of the Solar System's position in the galaxy. 


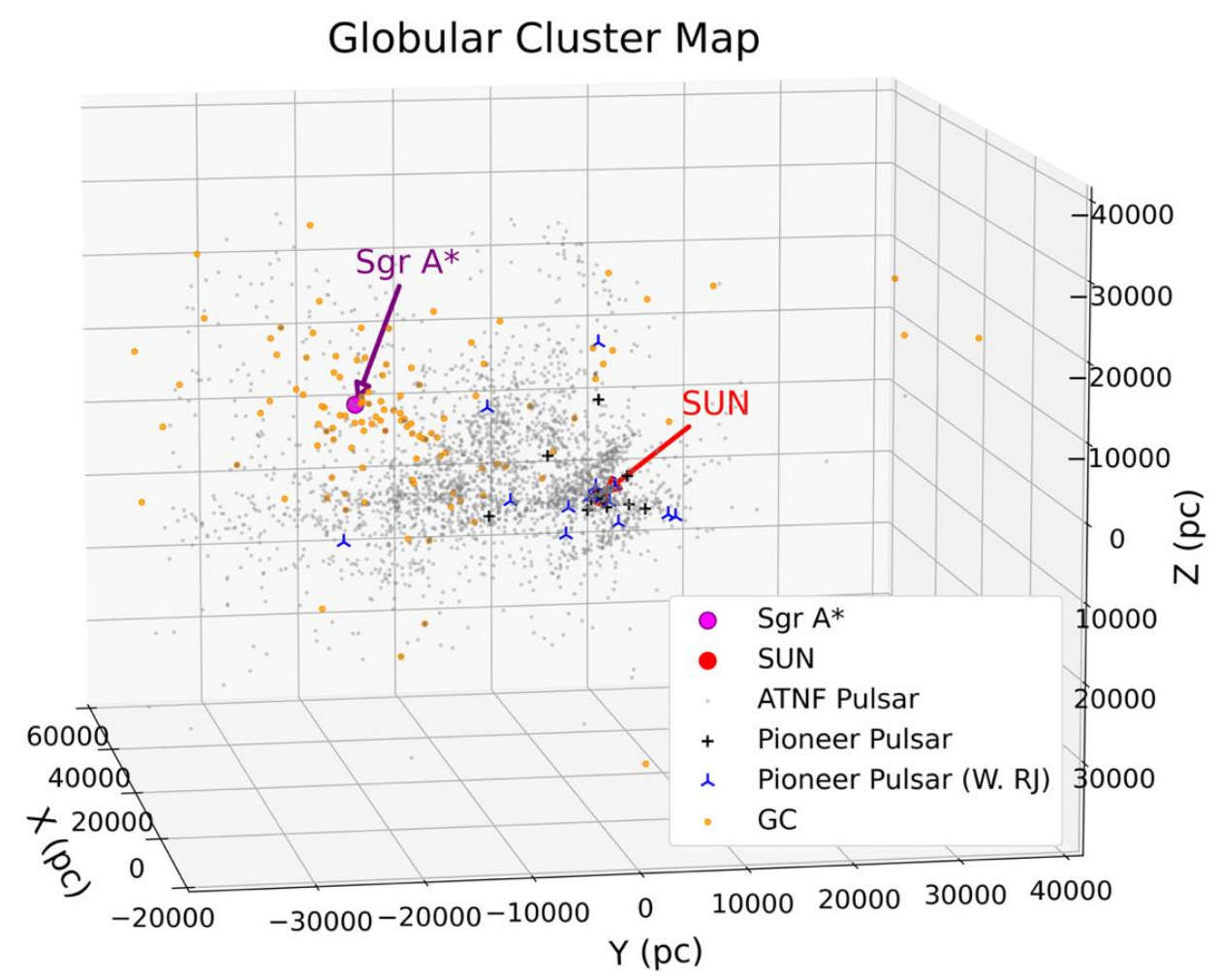

Figure 2.1: The blue point indicating Sgr $A^{*}$ represents the Milky Way's center. The red point is the Sun, to show our position in the galaxy. The dark gray points are the known globular clusters from https://heasarc.gsfc.nasa.gov/W3Browse/all/globclust.html by Harris (1996). The purple and orange points are known pulsars obtained from http://www.johnstonsarchive.net/astro/ pulsarmap.html, purple points are translated from the Pioneer plaque picture and the orange points are the corresponding pulsars from the ATNF database. The silver points are additional pulsars that have been discovered, these from the ATNF database.

\subsubsection{Time Stamp to Indicate the Timing of the Message Sender}

Because reference frames evolve over time, it is necessary to convey when our signal was sent as this is the initial condition for solving the corresponding reference frame. We are not sure when ETIs will receive the signal, so we must describe the departure time of our message via an absolute time scale: the age of the universe. The underpinnings of this approach to the time stamp include:

First, the cosmic microwave background (CMB) is the oldest electromagnetic radiation in the universe, dating to the epoch of recombination approximately 380,000 years after the Big Bang. The $\mathrm{CMB}$ has a thermal black body spectrum at a temperature of $2.72548 \pm 0.00057 \mathrm{~K}$, corresponding spectral radiance peaks at $160.23 \mathrm{GHz}$ [D. J. Fixsen 2009]. To describe this peak frequency, we could use the celestial $21.106 \mathrm{~cm}$-hydrogen $(\mathrm{H} \mathrm{I})$ line $(1,420,405,751.768 \mathrm{~Hz})$ as the base frequency, with the peak frequency of the CMB being 112.8 times this base frequency.

Second, according to the Friedmann equations the Hubble Parameter $H=\left[\left(8 \prod \mathrm{G} \rho / 3\right)-\left(\mathrm{kc}^{2} / \mathrm{a}^{2}\right)\right.$ $\left.+\left(\Lambda \mathrm{c}^{2} / 3\right)\right]^{1 / 2}$, which tells the speed of the universe expanding at a critical time, is also an indicator of the universe's age. In the Lambda Cold Dark Matter $(\Lambda \mathrm{CDM})$ model, $\mathrm{H}$ is decreasing over time and will approach a constant $\approx 57 \mathrm{~km} \mathrm{~s}^{-1} \mathrm{Mpc}^{-1}$. The present-day value is referred to somewhat misleadingly $\}$ as the Hubble constant, $\mathrm{H}_{0}=73.52 \pm 1.62 \mathrm{~km} \mathrm{~s}^{-1} \mathrm{Mpc}^{-1}$, as measured by the Hubble Space Telescope [Adam G. Riess et al. 2018]. It is also a way to describe our present-day value, 
albeit within these precision limits, and the Hubble parameter is a scalar so its value can be shown by the length ratio to its lower limit directly.

Third, the Globular Cluster Map contains the time information in itself - from the relative positions between each cluster the signal capture time can be discerned. If the ETIs have the equivalent of an ephemeris, which is likely if they already possess the technology to build radio telescopes, or if they can perform simulations to recover this map from the globular cluster map of their time, they could back calculate the signal sending time. With the CMB-based method, we can include the necessary information content we want in the BITG message while reducing the overall bit size of the signal.

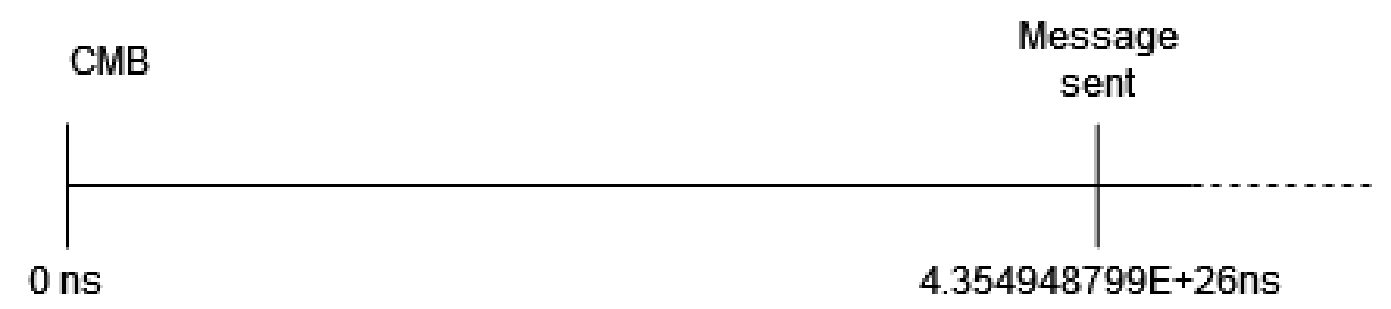

Figure 2.2: The time when this message be sent can be indicated by using the spin flip transition of the $\mathrm{H}_{2}$ molecule as the metric for time.

Finally, a more precise way to indicate when the message was sent would be to use the spinflip transition of neutral hydrogen in combination with a timeline and the CMB. As previously mentioned, the spin-flip transition of the $\mathrm{H}_{2}$ molecule was used as the metric of length and time on the Pioneer and Voyager messages. The frequency of the hydrogen atom electron is about $1,420.406 \mathrm{MHz}$, which corresponds to a period of $0.704 \mathrm{~ns}$ and a vacuum wavelength of 21.106 $\mathrm{cm}$ (length). Using the CMB as a starting reference on the timeline, calculation of the time the message was sent out is possible using the math operators explained earlier. On the timeline the origin of the CMB (13.8 billion years ago) would be 0 and the time when we write this sentence today, as measured from this beginning, would be $\sim 4.354948799 \times 10^{26} \mathrm{~ns}$ (Figure 2.2). Thus, if giving the age of the universe directly in terms of hydrogen spin flips, our time is $\sim 6.19 \times 10^{26}$ periods. Using the math operators, the ETIs would be able to calculate the time interval between receiving the message and when the message was actually sent from Earth. Note that given the criticality of the timestamp, any subsequent transmissions to the BITG message which humanity sends forth will require an update of the timestamp in the aforementioned terms.

\subsection{Optimal Date and Time for Sending the Message}

\subsubsection{FAST Observable Field}

Located in Pingtang, Guizhou, China, FAST is the largest radio telescope in the world. Due to its huge aperture and extremely high sensitivity, it is expected to be used in the search for intelligent life off the Earth. The FAST's design is similar to that of the Arecibo Observatory. The main mirror is built taking advantage of the local karst terrain and can be slightly deformed under the control of the actuator. target pointing and tracking are realized by the movement of the suspended feed source bin. The declination of the observable sky area of FAST is limited by the geographic location of the telescope and the movement range of the feed source. FAST is located at Lon $106^{\circ} 51 \not 24.0^{2} \mathrm{E}$, Lat $25^{\circ} 39 \not 10.6^{2} \mathrm{~N}$, the achievable pitch angle of the feed is between $40^{\circ}$, thus the corresponding sky declination is between $-14.6^{\circ}$ to $65.6^{\circ}$ [https://nadc.chinavo.org/s/2019/20190115_notice/f2.pdf]. 
The observational sensitivity depends on the temperature of the receiver. When the zenith angle is greater than $26.5^{\circ}$, the receiver is illuminated by the ground, the temperature rises, and the illumination area is less than the maximum that would be defined by the full $300 \mathrm{~m}$ diameter of the dish (Figure 2.3). The resultant sensitivity thus begins to decrease, so accordingly it is recommended to choose a source within $30^{\circ}$ of the zenith angle.

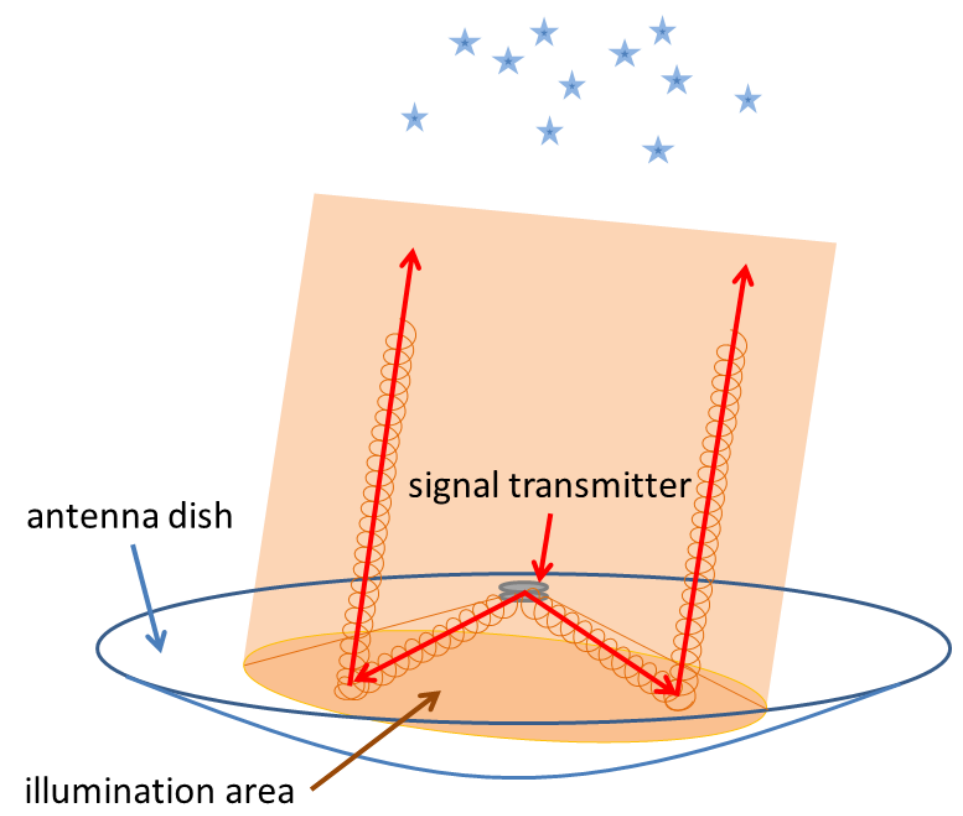

Figure 2.3: A diagram illustrating the FAST antenna dish and illumination area for receiving and transmitting signals.

The observable field delineated by the parameters of FAST is shown in Figure 2.4, presented on a sky map drawn using galactic coordinate system. The dark gray area is the unobservable area, and the light gray area is the area where the zenith angle is greater than $30^{\circ}$. To obtain the highest possible observational sensitivity, these areas need to be avoided. The red lines in the figure correspond to rings concentric to the galactic center at distances of 2,4 , and $6 \mathrm{kpc}$, which are considered the most likely locations for finding extraterrestrial intelligence [Cai et al. 2021]. To match the observable sky area, we can aim within the galactic plane to between $27.31^{\circ}$ and $30.00^{\circ}$ of the galactic longitude, where because of the projection of the potential ETI ring, higher densities of stars are found in this direction, with more potential ETI receiver targets.

As well, in order to facilitate the observation of our signals by ETI, it is necessary for our signals have the highest possible contrast to avoid dim or disappear in the sunlight. To minimize radio interference the separation angle between the Earth and the Sun should be as large as possible, which can be approximated by the Earth, Sun, and the location of ETI being at a relative position of $90^{\circ}$, maximizing the contrast between the radio signal and the sky background. Simultaneously, in order to reduce absorption by the Earth's atmosphere and have the longest tracking time, it is necessary to make the launch target reach the position with the smallest zenith angle. It is calculated the dates when the Earth-Sun-ETI target region will reach this relative position of $90^{\circ}$ is around March $30^{\text {th }}$ (orange square) and October $4^{\text {th }}$ (orange triangle) each year, and the right ascension of the observation target needs to reach $-80.5^{\circ}$ (blue square), the center time is 07:05 
and 18:41 on the corresponding dates, Beijing local time. When FAST fixes on the maximum zenith angle of $30^{\circ}$, the maximum tracking observation time is about 2 hours, that is, the target can be in the center to transmit or receive signals within 1 hour before and after the aforementioned times of day.

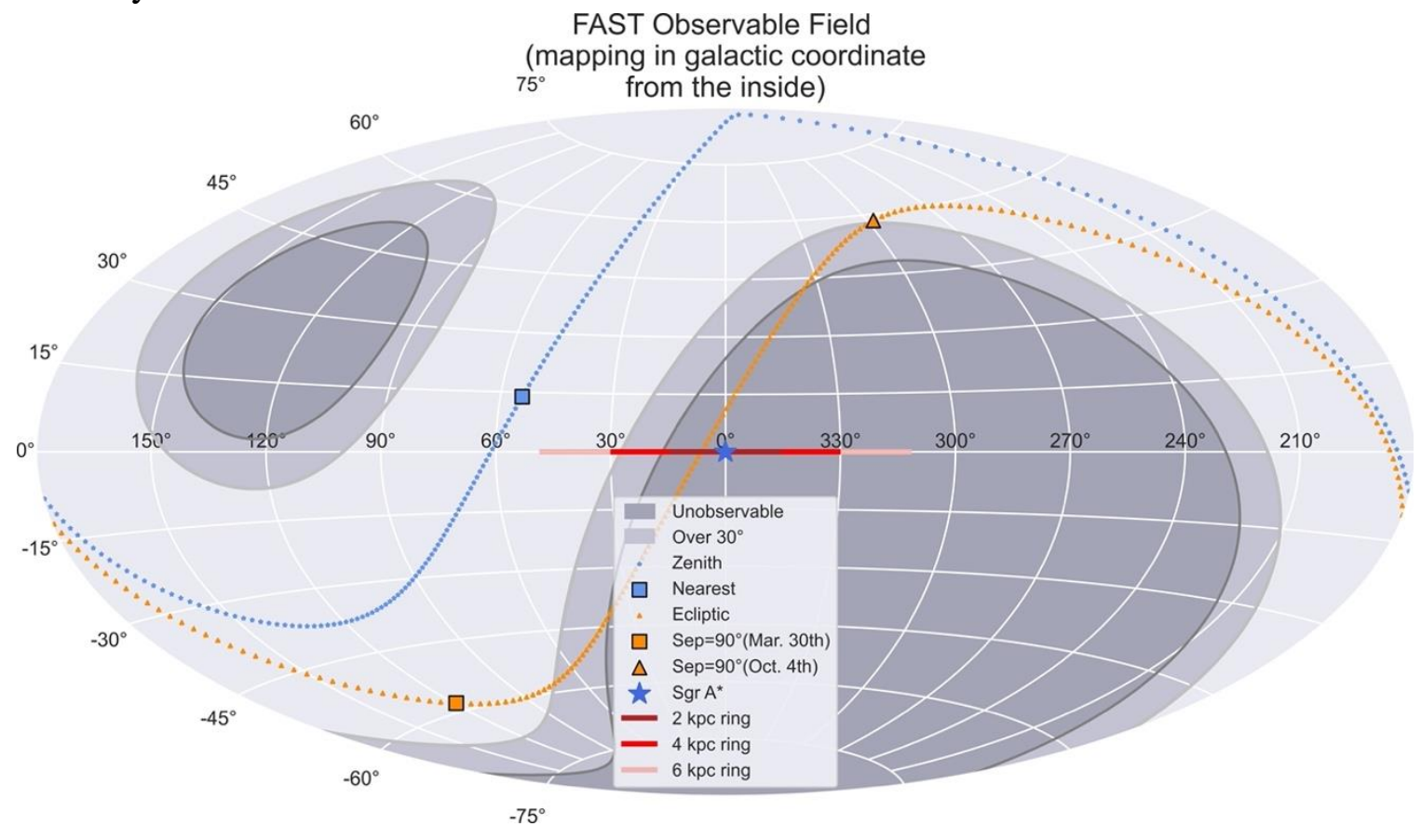

Figure 2.4: Observable Field of FAST

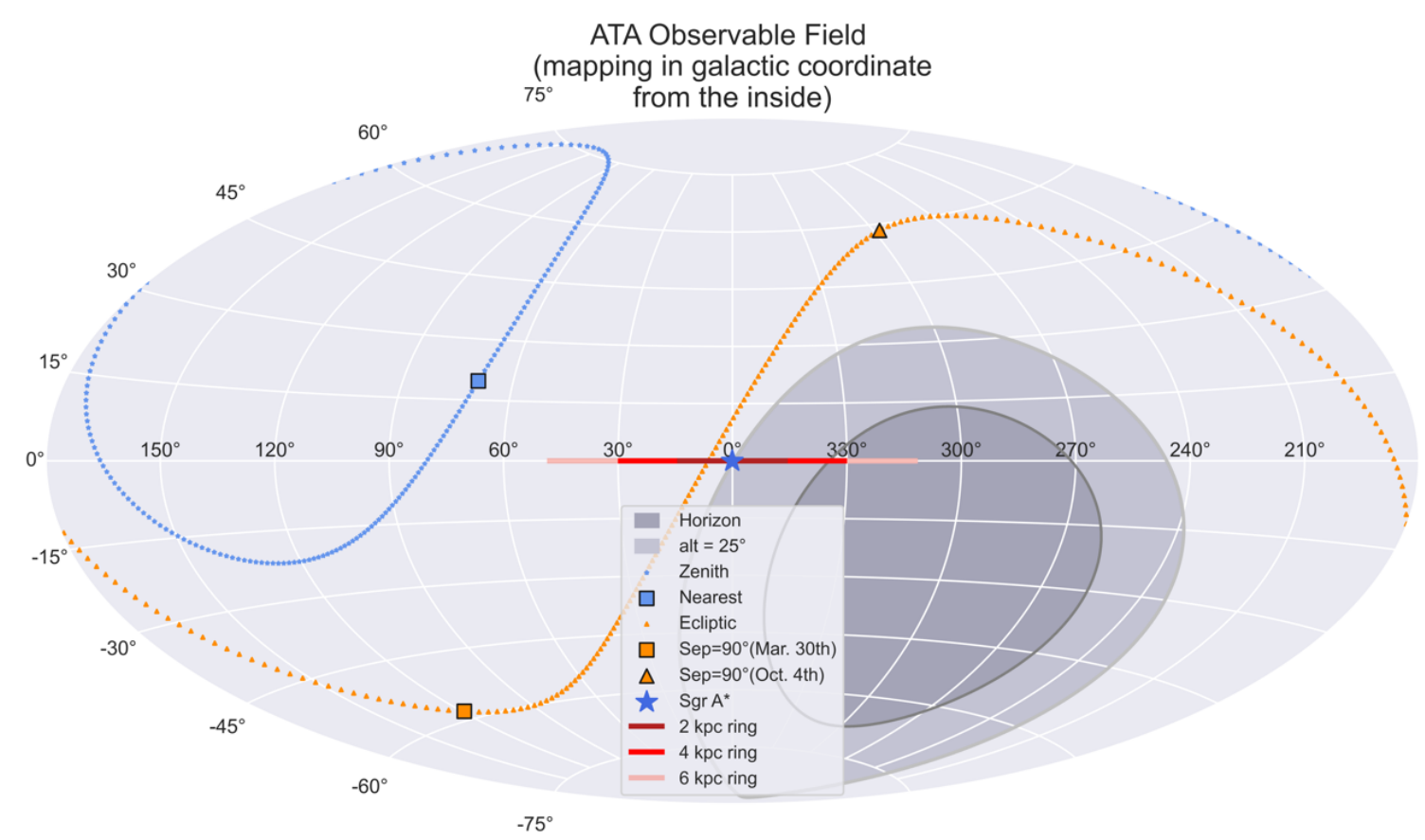

Figure 2.5: Observable Field of ATA. 


\subsubsection{ATA Observable Field}

The ATA's coordinates are Lon $121.47^{\circ} \mathrm{W}$, Lat $40.82^{\circ} \mathrm{N}$. Figure 2.5 shows the sky map drawn relative to the ATA location in the galactic coordinate system. Due to the ATA's mount being movable, the telescope could observe all targets in the northern sky, and part ways into the southern sky with the horizon being the limit of the observable field. We assume the lowest observable altitude of the ATA is $25^{\circ}$, thus the southern target's declination must be larger than $-15.8^{\circ}$. The separation between the target and the Sun is not affected a given transceiver's location on the Earth, thus we use the same dates derived in the FAST calculations for the ATA: March $30^{\text {th }}$ and October $4^{\text {th }}$. To obtain the highest sensitivity, the target should reach the position where it is at its nearest point to the zenith, the corresponding time (UTC) would then be 14:10 for March $30^{\text {th }}$ and the 01:50 for October $4^{\text {th }}$.

\subsubsection{Summary of the Best Times for FAST and ATA to Transmit Message}

The best dates and local times for FAST and ATA to send out the message to near the $4 \mathrm{kpc}$ ring concentric to the galactic center are summarized in Table 2.1.

Table 2.1: A summary of optimal dates and times for FAST and ATA to transmit the BITG message.

\begin{tabular}{|c|c|c|c|c|}
\hline Date (UTC) & $\begin{array}{c}\text { Separation angle of } \\
\text { Sun-Earth-Target }\end{array}$ & Telescope & $\begin{array}{c}\text { Best Observe } \\
\text { Time (UTC) }\end{array}$ & $\begin{array}{c}\text { Maximum } \\
\text { Altitude }\end{array}$ \\
\hline \multirow{2}{*}{ Mar. 30 } & $\sim 90^{\text {th }}$ & FAST & $23: 05$ & $59.53^{\circ}$ \\
\cline { 3 - 5 } & \multirow{2}{*}{${\text { Oct. } 4^{\text {th }}}^{*}$} & ATA & $14: 10$ & $44.35^{\circ}$ \\
\cline { 3 - 5 } & $\sim 90^{\circ}$ & FAST & $10: 41$ & $59.53^{\circ}$ \\
\cline { 3 - 5 } & ATA & $01: 50$ & $44.35^{\circ}$ \\
\hline
\end{tabular}

\subsection{Coding Design}

\subsubsection{Coding Methodology}

As detailed in section 3, below, the BITG message is comprised of 13 parts that consist of approximately 204,000 effective binary digits, or 25,500 bytes, overall. To generate the particular set of matrices required, composed only of "zeros" and "ones" which will display as a visual message, the coding is done by specifically constructing from position dependent elements to reach the desired visual effect. The codes in the MATLAB ("MATrix LABoratory") programming language are given in the online attachment. Only a summary is given in this section.

There are three ways to build up a matrix working behind lines of code. The first and generally simplest way is to "weave" a matrix row by row typing in zeros and ones (or a continuous series of them as one type of shortcut). A manually typed-in matrix is assigned to a variable and thus the workspace in this case. Such a method is used at the beginning of the coding and is also the most independent option since nothing is introduced to the file other than the codes itself - i.e., it does not rely on any external information. The overall coding can, however, be lengthy because each row of the matrices corresponds to a line of code.

The second way is to directly "copy" an existing image to reproduce the visualization of that image via coding. A locally pre-stored image is imported into the workspace and then analyzed in an assigned resolution and binarized into matrices corresponding to red, green, and blue lights. The matrix of the most significant color of light is then chosen to swap zeros and ones so that black and white is correspondingly flipped over, resulting in two-toned (i.e., black \& white) images as 
objectives. This method is useful and generally straight-forward for reproducing past message contents and is assisted by subroutines for manual editing to correct any detail that is missed by the automated conversion. The code for editing consists of a "for loop" with iterations that can be changed after each run for different numbers of matrix elements to be changed. The capability to read the location (coordinate) of the cursor, round the coordinate into whole numbers, assign the corresponding element pointed to by the cursor with a "zero" or "one", and display the change immediately thereafter are all contained within the "for loop".

The third way is to fill out the blank by blocks - adding contents from other matrices by replacing blocks of zeros with those contents. The first step is to construct a blank (all zeros) matrix with desired size given. The next step is to select a rectangular area within the matrix and assign values copied from other matrices to those elements. The digitized size of the source information matrix and that of matrix being assigned to code that information must be equal to perform a successful operation. On the whole, the matrix being constructed is composed of multiple rectangular information sub-matrices, like a puzzle but with the pieces in different sizes and able to overlap on each other to overwrite the previous value(s) assigned to those locations.

The final product of the matrix generating codes should be the matrices stored in the form of spreadsheet files, this so manual operations only exist when actually generating the message - i.e., the generating codes are tools to reach the product rather than the skeleton of the product itself. When sending a message, a synthesizing code is used to read all the Excel files in the same folder and organize them into a single message ready to be sent. Execution of the synthesizing code is automated as long as it is in the same folder with the Excel files mentioned above, noting the matrix generating code is not necessary at this step.

\subsubsection{Location Stamp Codes}

Globular Cluster Map

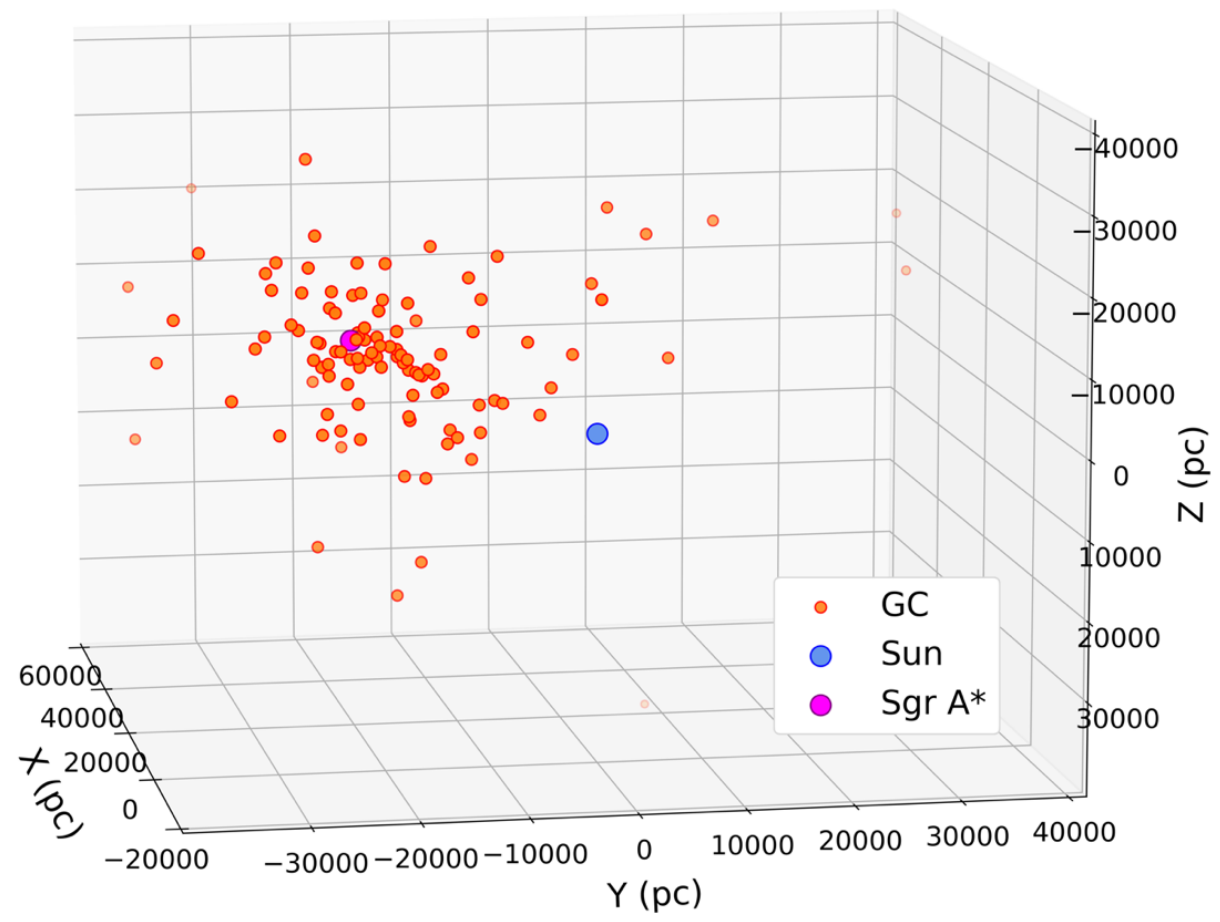

Figure 2.6: Globular Cluster Map for pinpointing the location of the Solar System. 
To send the globular cluster map via radio telescope, the first challenge is how to convert the map to a radio signal with confidence the signal can, if and when received, be easily decoded. As previously mentioned, the Pioneer and the Voyager robotic deep space probes chose a picture message. In principle, we can use the same method. However, to enhance the fidelity of the received signal while maintaining the full content of the message, the size of the message should be minimized. If we were to put a location picture analogous to that of the Pioneer plaque into binary, the size would be larger than $10^{5}$ bits and with some loss of accuracy. Alternatively, sending the coordinates directly decreases the message size. To accomplish this, we convert the globular cluster position data from the spherical coordinate system to the Cartesian coordinate system in lightyear units, constructing an $80,000 \times 80,000 \times 80,000$ lightyears cube, see Figure 2.6. Note that this does exclude 37 distant globular clusters. Then, calculating the differences of the $\mathrm{X}$, $\mathrm{Y}$, and Z-coordinates from their respective axes for the remaining 120 clusters, we get the minimum differences of these three axes to about 1 lightyear, which defines the limit of approximation to 1 lightyear for all cluster coordinates. Thus, the range of the coordinate is 0 to 80,000 lightyears with a 1 lightyear resolution. Given the assumption that for technical civilizations binary is a fundamental of known mathematics, the coordinates when converted to binary signals result in a total number of globular clusters of 120 and, along with the Sun, the size of that portion of the message is $17 \times 3 \times 121=6,171$ bits. To avoid possible confusion with interpreting the signal, some separators, the Sun symbol, and the line ending symbols are also necessary. These symbols can be used elsewhere in the overall message to keep it as short as possible and further reduce the potential for confusion. The details of the location stamp codes are given in the Appendix A.

\section{Results and Discussion}

The updated BITG message is a set of radio signals carrying basic information about humanity and our place and time in the Milky Way galaxy. The full image of the message is given in Appendix B. It consists of 13 parts that encode the following from the top down in the image.

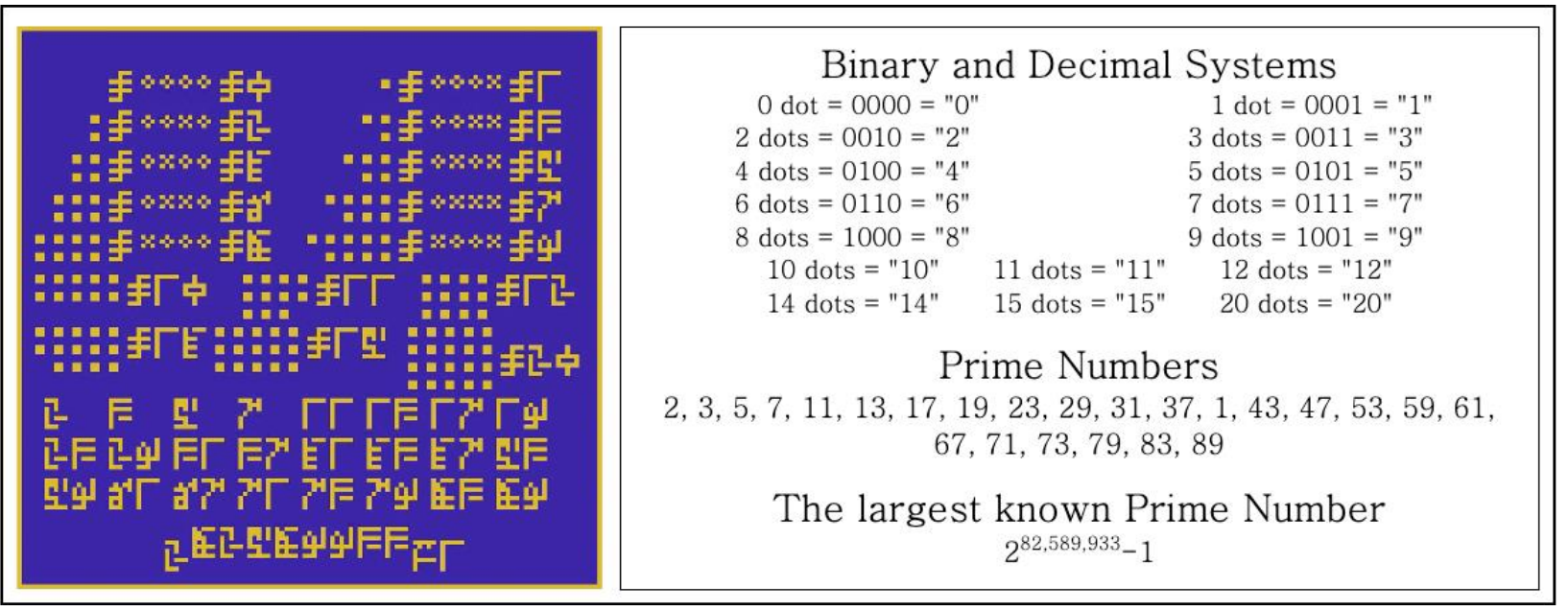

Figure 3.1: Pictural representation and explanation of the BITG message, page 1.

The message begins with a header as described in section 2.1, this to call the attention of any recipients by the use of prime numbers. The first page of the BITG message must contain information both universally recognized but also imperative to the understanding of the rest of the 
message. As the message is sent in binary, recipients will very likely understand fundamental elements of mathematics, physics, and chemistry. Hence, the message begins with a binary representation of our numbering system, communicating the human base-10 counting system.

Page 1 of the message, as shown in Figure 3.1, describes the numbers 0 through 9 in both the corresponding number of dots and binary with a pictural representation that will be used throughout the rest of the message. The message utilizes dots to represent numbers by simply assigning $\mathrm{x}$ number of dots per number, with $\mathrm{x}=$ the number. Following digits 0 through 9 , the message explains the base- 10 system with 10 dots $=10$, extending into 11 dots $=11,12$ dots $=12,14$ dots $=14,15$ dots $=15$, and 20 dots $=20$. This unambiguously represents base-10 and makes the communication to follow comprehendible as it explains how to read numbers greater than 9, crucial for the understanding of distance, time, and mass later in the message. The page ends with a list of the prime numbers between 2 and 89, this as previously mentioned, to confirm the intelligent origin of the BITG message noting again that prime numbers are a clear indicator of life given that natural cosmological processes are highly unlikely to result in prime number sequence generation (Sagan, 1975).

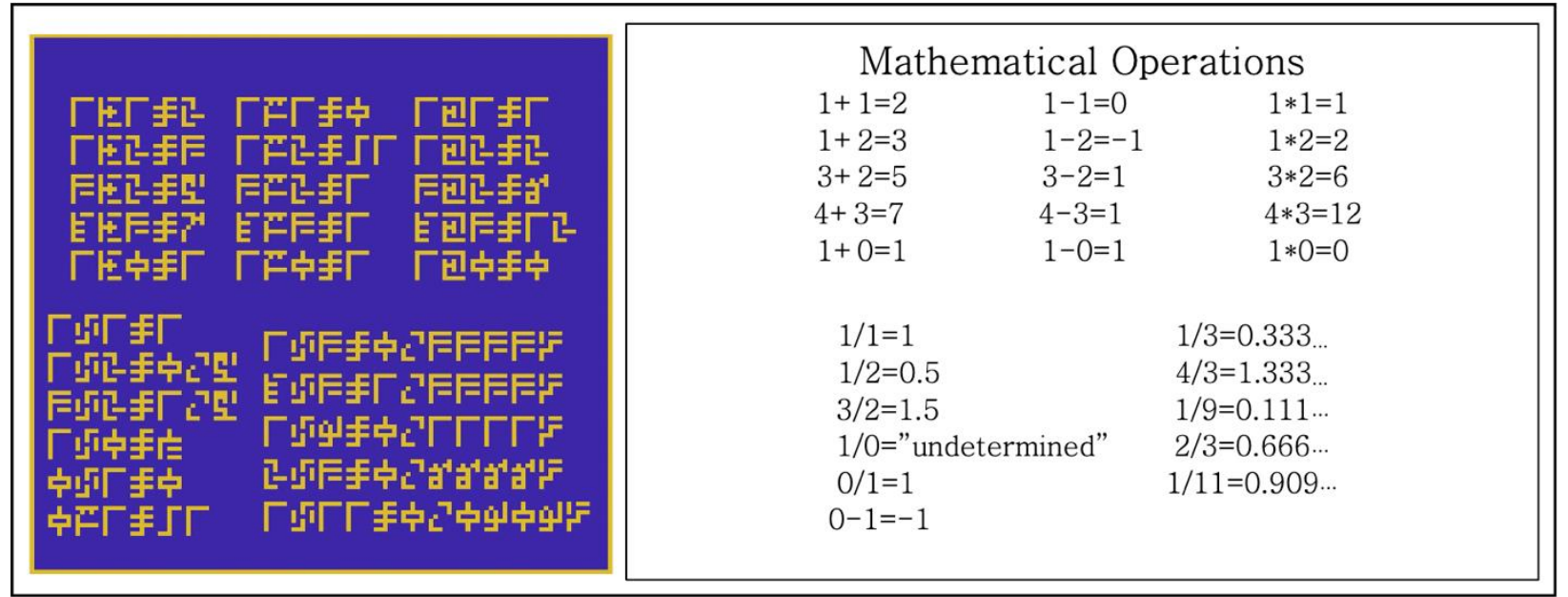

Figure 3.2: Pictural representation and explanation of the meaning in the Message, page 2.

A logical evolution of information would be the progression of mathematics: basic operands like addition, subtraction, multiplication, and division; additionally, the more advanced concepts connected to the idea of division are included in continuity and undetermined. This information is at the core of our mathematics and is used later in the message to describe large values or more advanced concepts - or for any follow up messages sent in the future. This represents the first real test of the message's comprehensibility from human terms. Emulating, in essence, the progression of the human learning process, the page begins with basic addition, subtraction, and multiplication, starting with $1+1$ and $1-1$ being the simplest operations in our mathematics, and $1 * 1$ being the simplest multiplication possible. These basic operand rules and their special rules are taught in a logical progression such as we learn in early grade school. For example, on the 5 th line of the page the identity rules of addition and subtraction and the zero rule of multiplication are introduced, these being essential mathematical properties that any intelligent extraterrestrial should logically possess an equivalent. The more complex idea of division (from the human perspective) especially with irrational numbers - is introduced last, along with the continuity symbol, the concept of undetermined numbers, and the identity rule of division. Page 2 of the message, as 
shown in Figure 3.2, builds off the previous introduction to math and serves as a good segue into the more complex ideas to follow.

The first two pages of the BITG message are designed as descriptions of human interpretation of universal concepts. The third page begins by introducing another such concept, exponentials. However, following the introduction of our numbering system for exponentials, the message begins to explore more distinctly human mathematics. Figure 3.3 is a pictural representation and content descriptor of page 3 starting with the most basic exponentials - again what should be a universal concept. For example, the reliance of Newton's law of gravity and Coulomb's law on squared distances are just two naturally occurring scientific examples of inverse square laws. While the logical next step is introducing the inverse of exponentials, i.e., roots, the BITG message instead takes a sensible pause to allow for the introduction of scientific notation. Designed very much around base-10 mathematics and nearly indispensable when quantifying astronomical measurements, scientific notation is described by introducing the powers of 10 and their use of a moving decimal point. Only after this does the message begin to describe roots, finally displaying as a simple example the most commonly appearing root and its numerical value: $(2)^{1 / 2}=1.41421356 \ldots$.

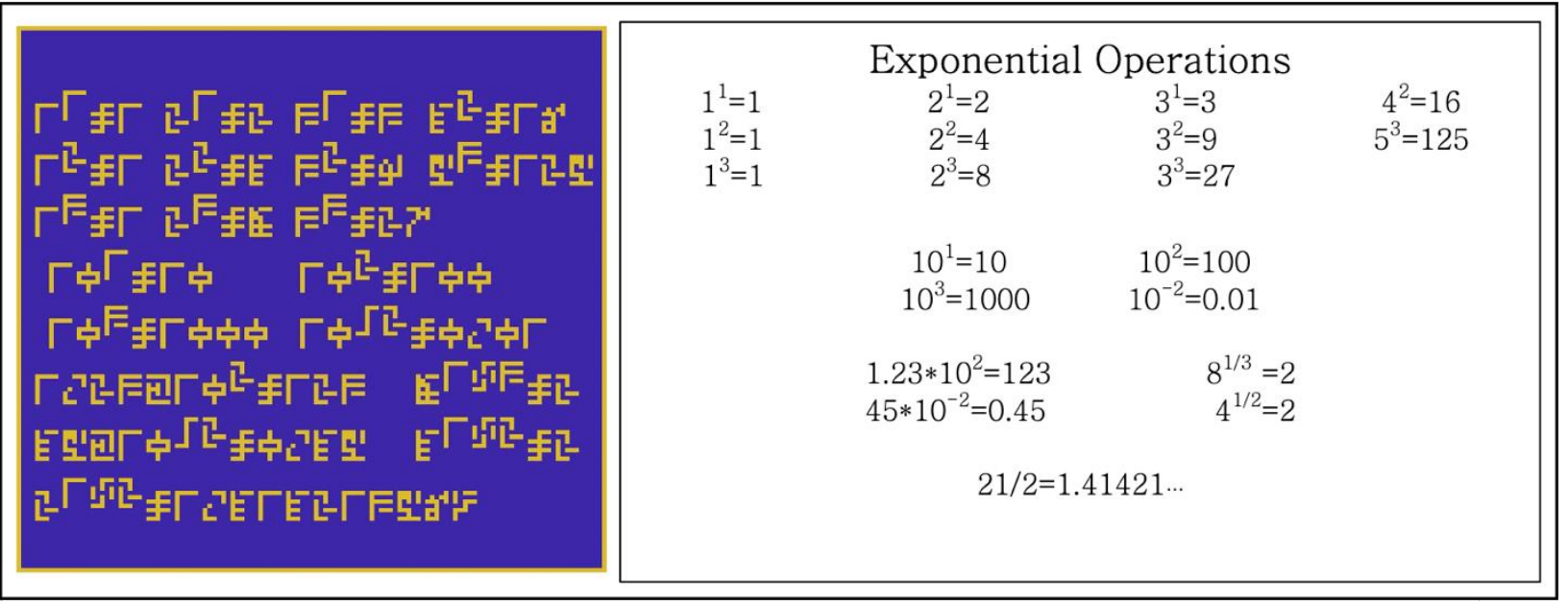

Figure 3.3: Pictural representation and explanation of exponents in the Message, page 3.

After exponentials and roots are described in the BITG message, the groundwork will have been laid for the final math concept of algebra to be introduced. Algebra is the basis for all advanced topics of science and every area of physics requiring expression in equation form. Thus, in order to establish a basis for scientific communication whether in this message or future messages, the topic of algebra must be considered foundational. In Figure 3.4, the fourth page of the message is presented - and here algebra is introduced. The page begins with basic algebraic functions used in solving for a single variable, in this case symbolically depicted as "a" with a question mark before the "a" to signify the focus on "a" as an unknown to be solved for and the "?" as a signifier of inquiry. The page progresses from single variable addition to single variable division, solving for "a" in each case starting with the simpler operations such as addition and subtraction before progressing to multiplication and division. Once the topic is properly exemplified, the message proceeds to use multiple variable algebra, describing the variable "a" in terms of two other variables: "b" and "c". Finally, the message ends the page with the idea of plotting variables against each other with a graph displaying a cubic function " $a$ " with respect to 
"b". As previously discussed, it is believed that mathematics is a universal concept that must accompany technological intelligence; thus, the first four pages of the message serve as our introduction for any ETIs into that realm of fundamental knowledge and the ways of human thinking.

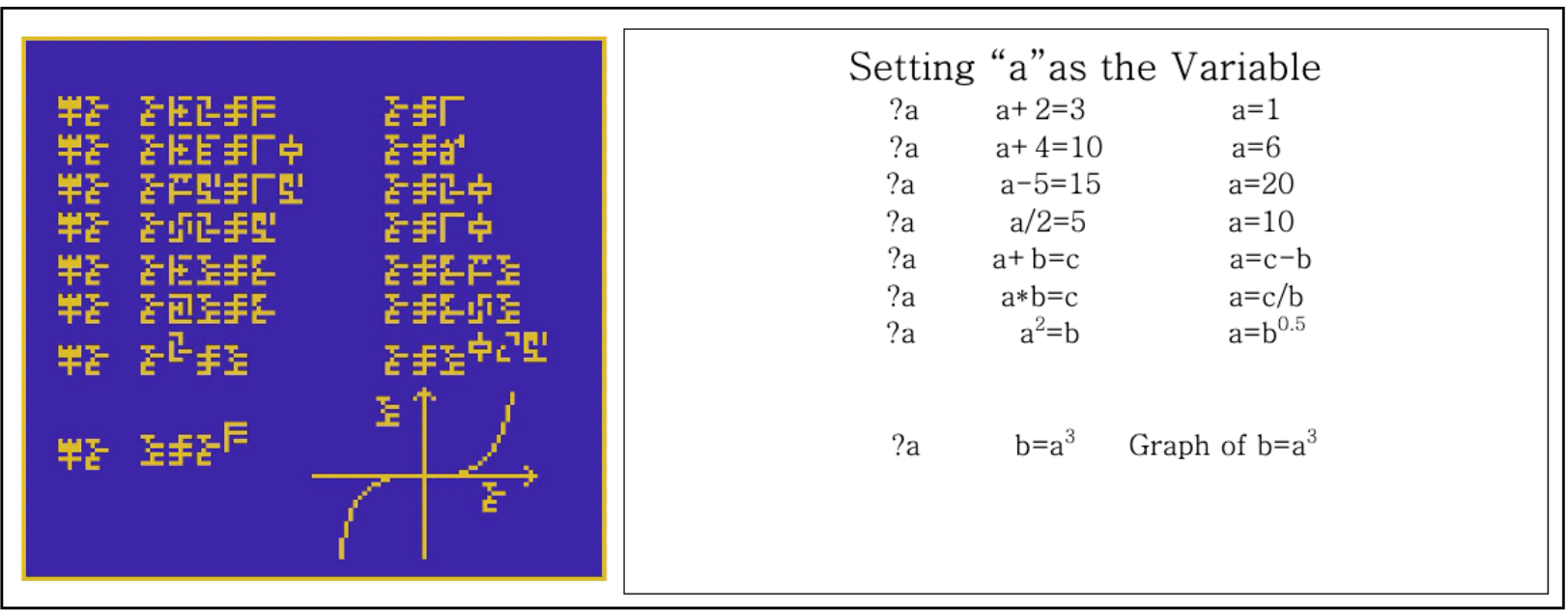

Figure 3.4: Pictural representation and explanation of algebra in the Message, page 4.

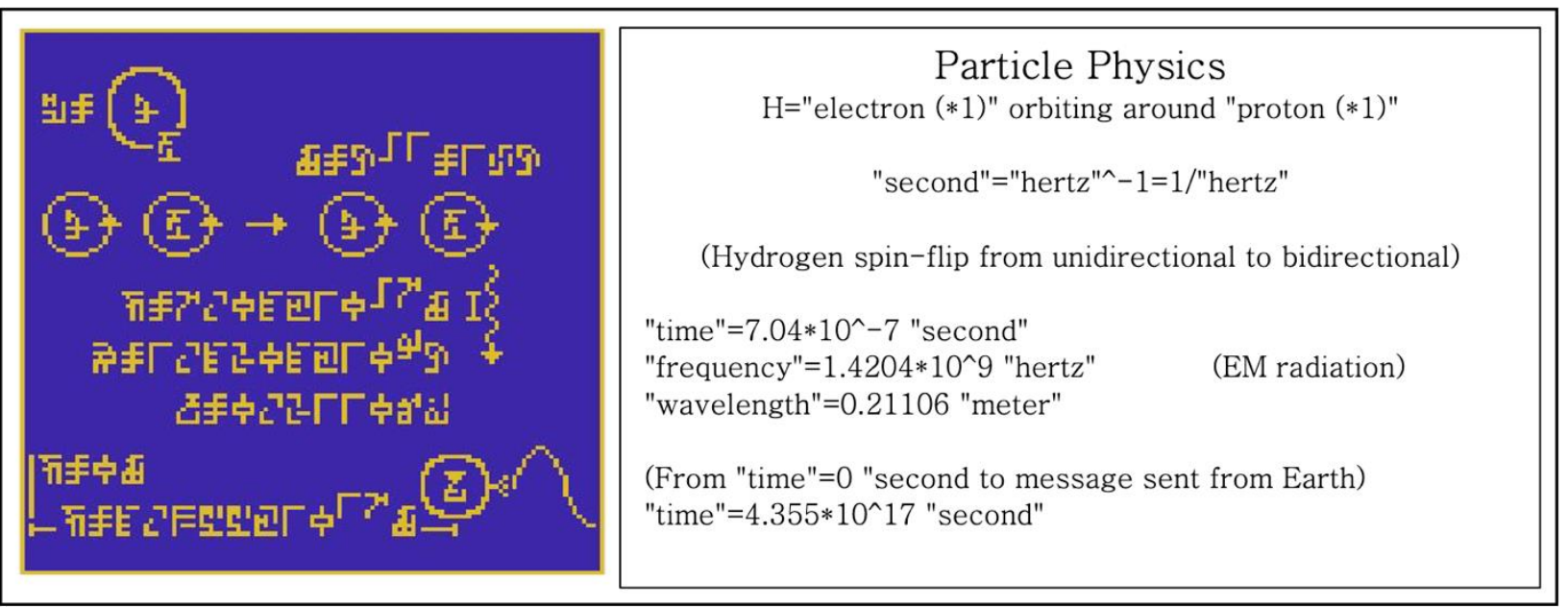

Figure 3.5: Hydrogen spectrum and hydrogen atom spin-flip transition in Message, Page 5.

While mathematical communication is of utmost importance, a message solely containing a description of our mathematics would tell any ETI very little about the humans which sent it. Progressing further, one such way of informing intelligent aliens about how we perceive the physical universe is by describing our base measurement units using a universal constant - but what constant? The hyperfine transition (the "spin-flip transition") of the hydrogen atom has been used before, and it makes sense to include here as well. $\mathrm{H}_{2}$ is, by far, the most common compound in the entire cosmos, so the properties of hydrogen should be well understood by a technological intelligence. By using the wavelength of the EM radiation generated by spin-flip transition and the period of that radiation, the BITG message includes in Figure 3.5 the definition of a meter and second. This page serves two main purposes in the greater scope of the message: the first as the 
initial descriptor of how we perceive and the second being an introduction to units such that the message can describe different and more complex physical ideas.

Mathematical means of communication and the spin-flip transition of the hydrogen atom can be described as another header of sorts - a preamble to the main message and necessary to understanding the main bulk of the message. The indicator, however, that the centerpiece of the message has begun is inclusion of a timestamp. The timestamp, as describe in Section 2.1.2, is an indication of when the message was created and sent - imperative to understanding the relative positioning of stars as they change across the thousands of years it could take for the BITG message to reach even the first of its possible destinations.

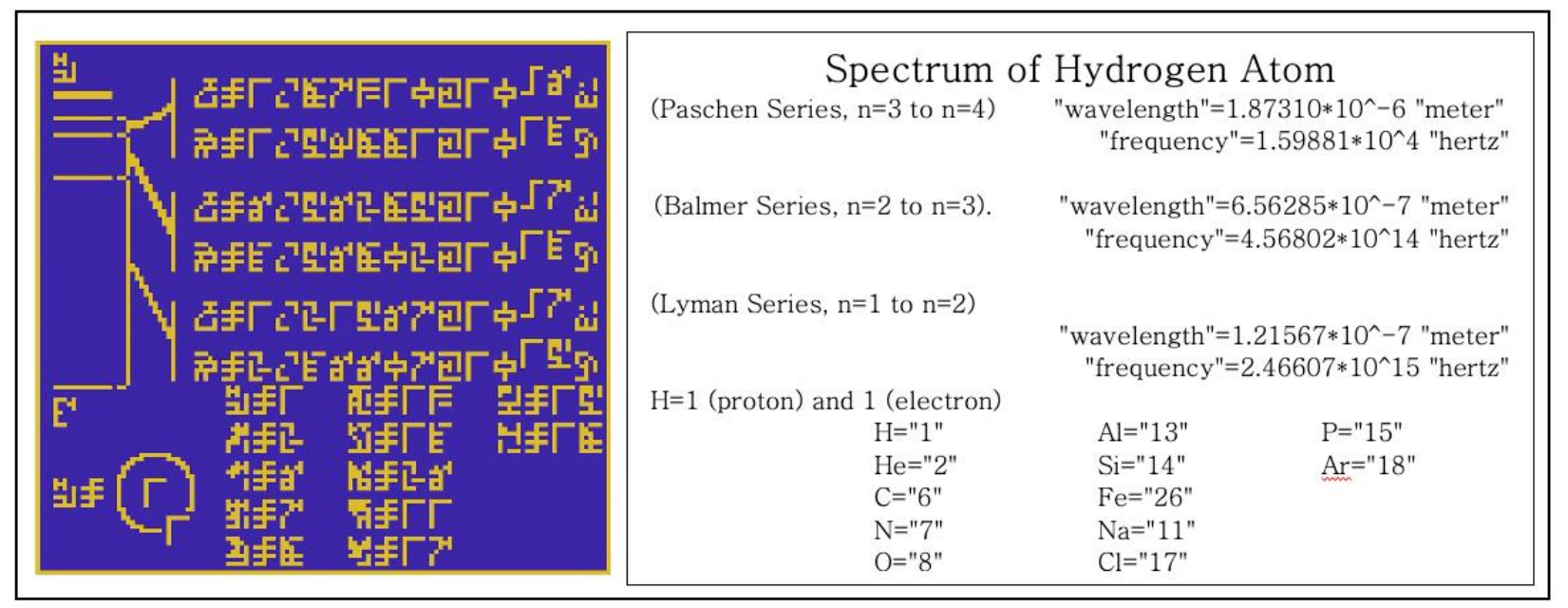

Figure 3.6: Most common elements in the Message, Page 6.

Although not the "official" header, a timestamp conveys the idea that the main content of the BITG message has not been seen yet but will soon follow. The final introduction of human interpretation of the galaxy is our understanding of elements, and what elements are most common in our experiences. By depicting the Hydrogen spectrum alongside "one" inside a circle marked by another "one" - seen in Figure 3.6 - we establish that Hydrogen is one, meaning that describing each element as its atomic number can be done for a series of the most commonly occurring elements. This would logically be of interest to any receiver as it helps to establish what humans most frequently perceive and interact with - and builds directly into the next page.

The BITG message is designed to follow as logical a progression as possible while adhering to essential basic communication protocols, all the while satisfying our relevancy test of including only such information as we would wish to receive from an ETI attempting communication with us. Hence, with basic math, physics and chemistry established the message progresses to the building block basis of all life on Earth - DNA. Deoxyribonucleic acid, being composed of commonly occurring elements, is the clear follow-on to the introduction of lower atomic number chemical elements. Not only is the information a logical progression of the chemistry introduced earlier, but the knowledge that humans, and all life on Earth, are carbon-based is a crucial point in describing our world and its inhabitants. Accordingly, the four bases of DNA - Thymidine, Adenosine, Cytidine, and Guanosine - are depicted in Figure 3.7 using the previously introduced chemical elements in Figure 3.6. 


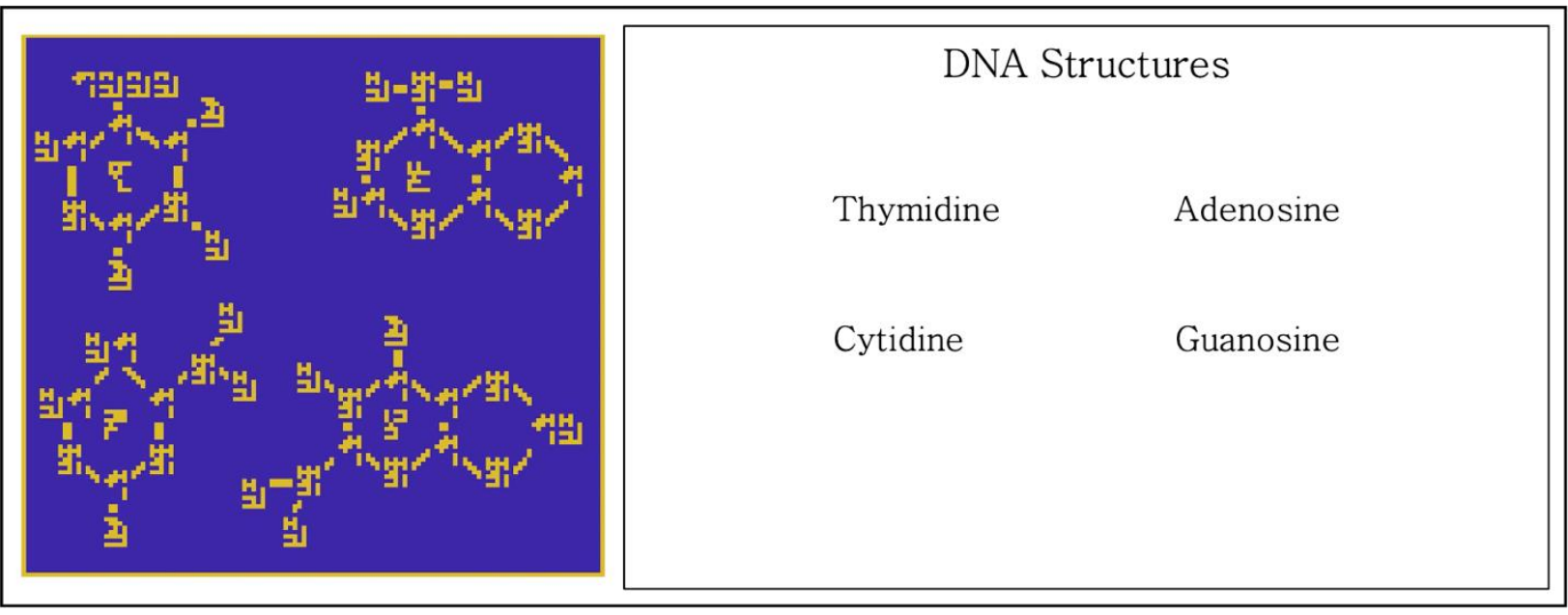

Figure 3.7: Pictural representation of DNA in the Message, Page 7.

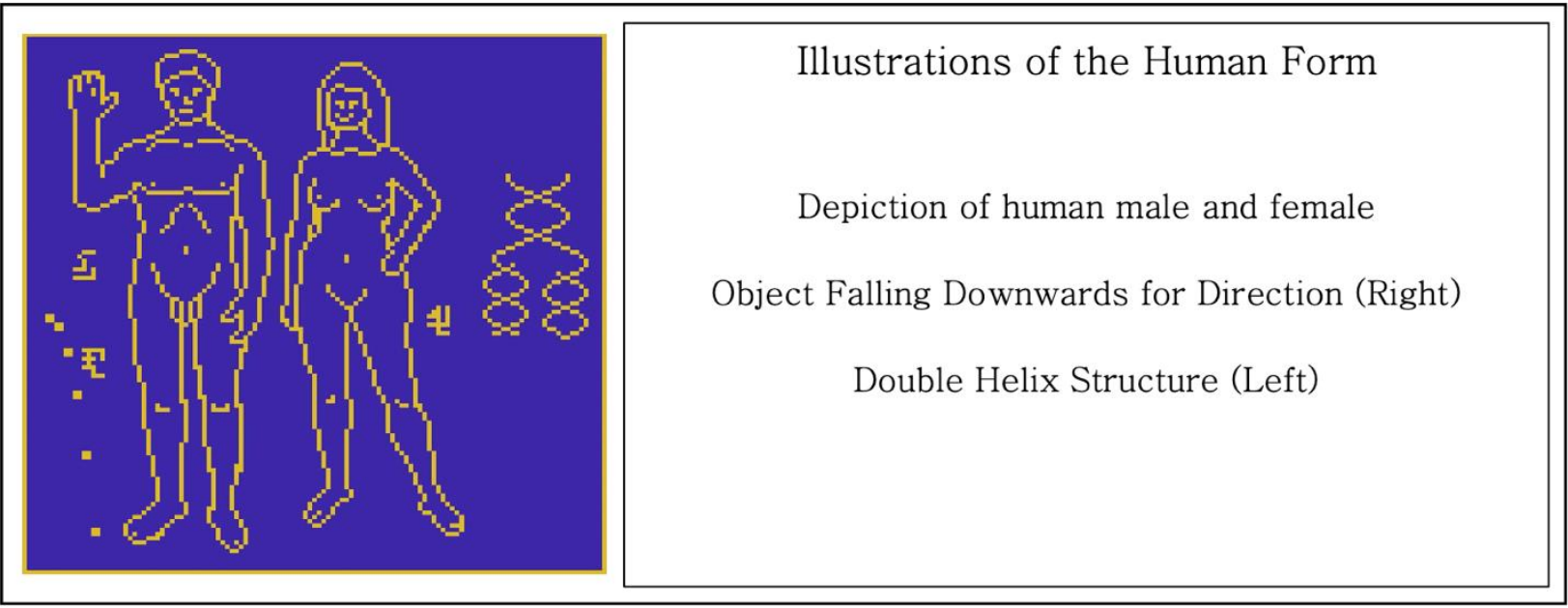

Figure 3.8: Pictural representation of humans and double helix in the Message, Page 8.

DNA's double-helix structure is key to its essential self-replication function, this ordered through its different base pairings, and hence the structure would logically be included in the page immediately after description of the DNA bases. Given DNA's central role in human biochemistry, depictions of male and female human anatomy should then accompany the double-helix structure. Hence, Figure 3.8 shows both the double-helix and an image of male and female humans along with the path of a falling object in the bottom left to help any recipient with orientation of the image. This page can easily be considered one of the most important parts of the message as a physical depiction of the senders of a cosmic message would certainly be of compelling interest.

With information about the senders complete, the BITG message progresses to defining the location of the Solar System within the Milky Way galaxy. This is done using progressive location mapping to find a particular point: one starts from the most broadly known region containing the point of interest and from there narrows down via references through ever smaller scales to that specific point. With this approach in mind, the message goes on to describe our host star and its planetary system in the Milky Way with a map of the Solar System. Figure 3.9 is a representation 
of this in simplified form - a guide to our Solar System's basic configuration with an indicator for the Earth.

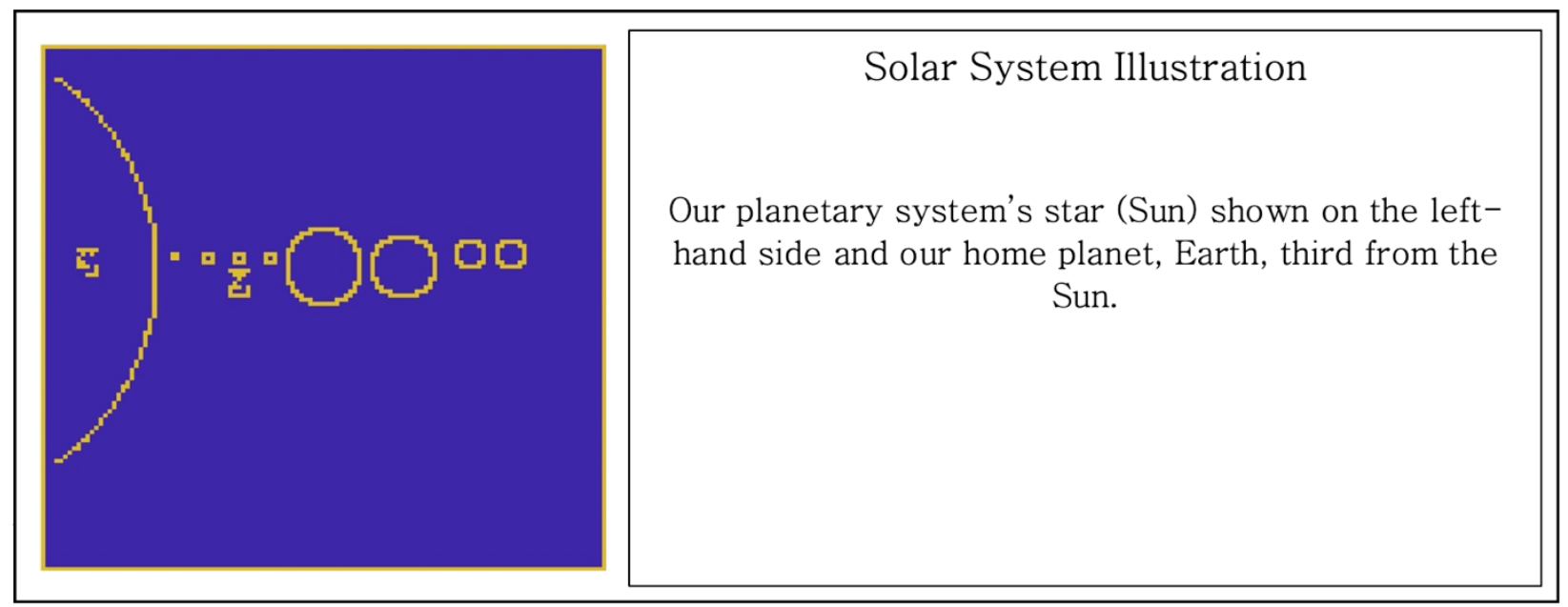

Figure 3.9: Pictural representation of Solar System with indicator to Earth in the Message, Page 9.

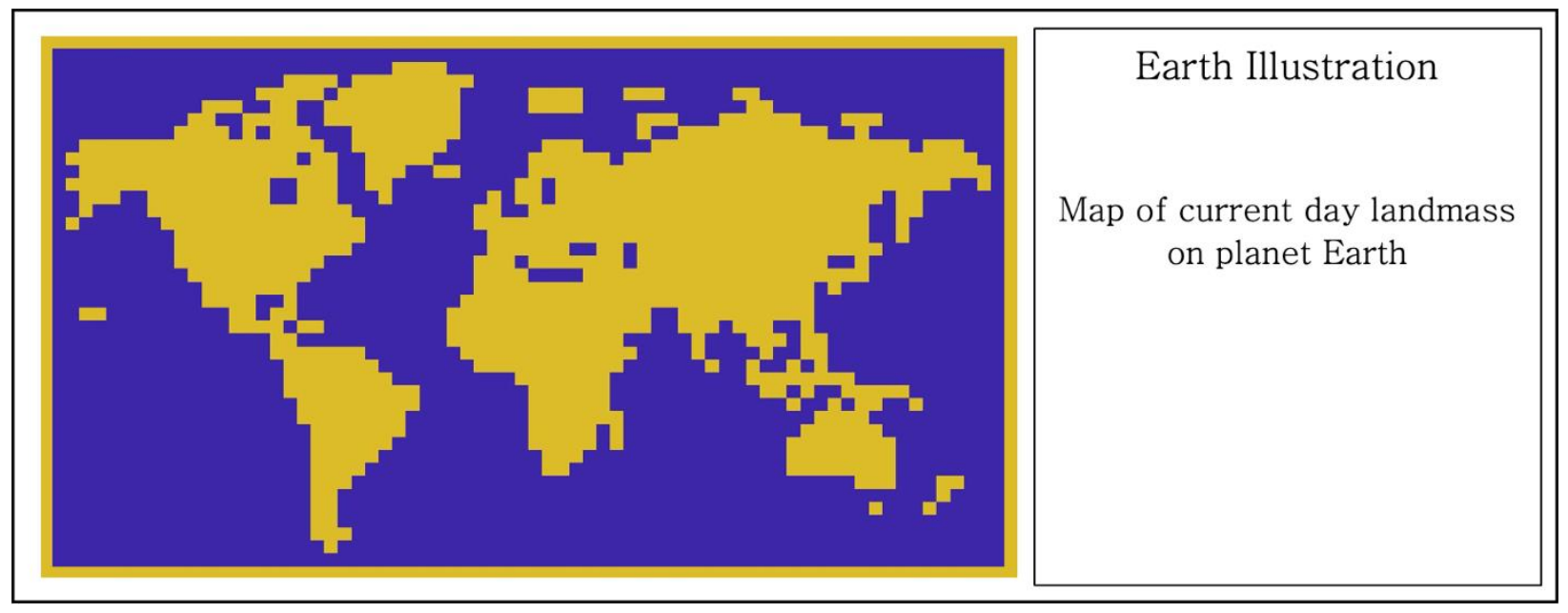

Figure 3.10: Map of the Earth in the Message, Page 10.

In the same vein of thought, the next page of the message contains a map of the Earth. In Figure 3.10, we have reached the level of finest detail in the BITG message's multi-stage depiction of our galactic address - a basic mapping of our home world. This information helps to make Earth more distinctive and provide any recipient the opportunity to understand the Earth's surface as seen from near space. Additionally, after knowing what the Earth looks like, it follows that a recipient would be curious as to the Earth's general composition - i.e., what composes the Earth's atmosphere and its crust. Taking the Earth surface map a step further, one might even suppose an intelligence hypothesizing of how our largest land masses might lend themselves to a localized history that included the rising and falling of empires while extensive coastlines could imply societies built more around commerce. It should be noted that such speculation is necessarily from a human perspective and may well not apply to intelligences which arose under other suns. 
This train of thought leads directly into Figure 3.11, a breakdown of the most commonly found elements in and on the Earth, serving not only as key facts for ETI planetary scientists but also providing valuable insights to our way of life and advancements of our civilization to near mastery of our world.
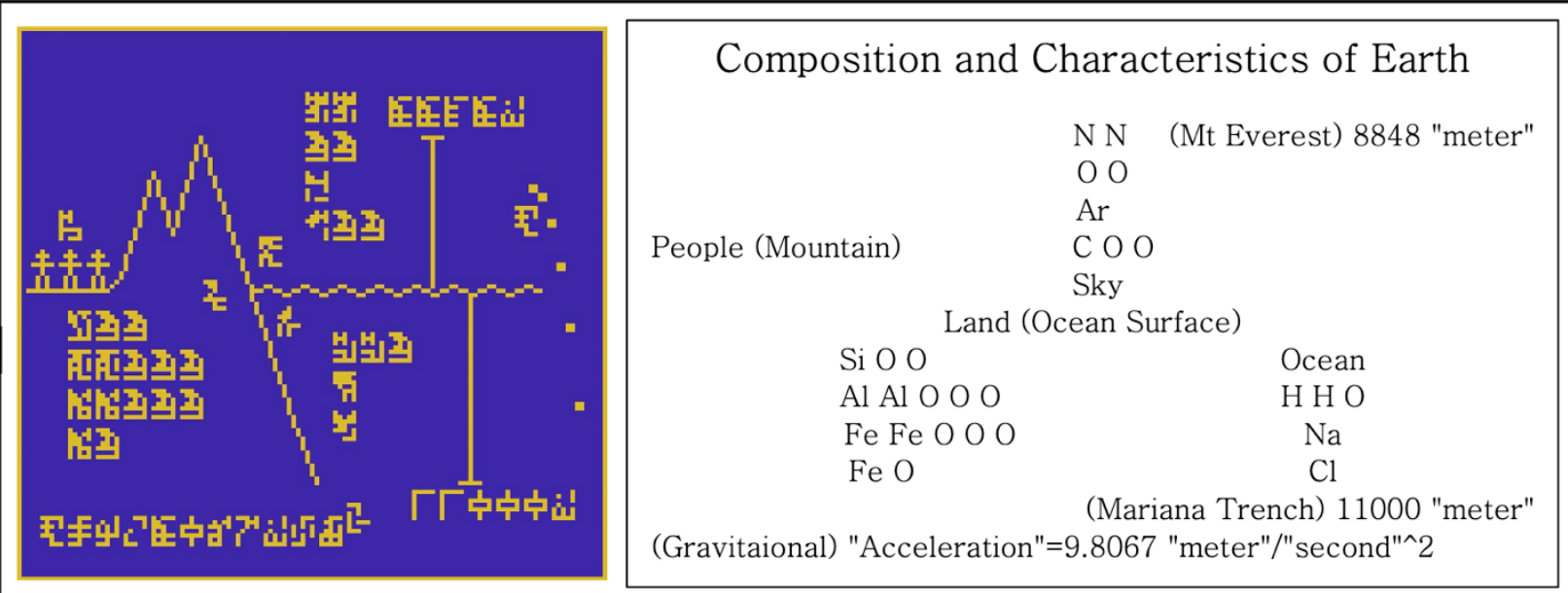

Figure 3.11: Earth compositional breakdown in the Message, Page 11.

Finally, the goal of the BITG message was always to make contact and hopefully begin a dialogue with ETIs located within the Milky Way. This mission to initiate a conversation of course requires reciprocal action on the ETIs' part - that is, to send a return message. Figure 3.12 depicts two telescopes with an electromagnetic wave, at the frequency of the BITG message, propagating between them suggesting we would expect to receive replies from ETIs in that same frequency. This is an invitation to reply with a message to us using a radio telescope apparatus of their own. The BITG message ends on this page as an open invitation to any ETIs that may receive it to respond to the sender - expanding both civilization's scientific knowledge but more importantly, establishing for the residents of these worlds that they are not alone.

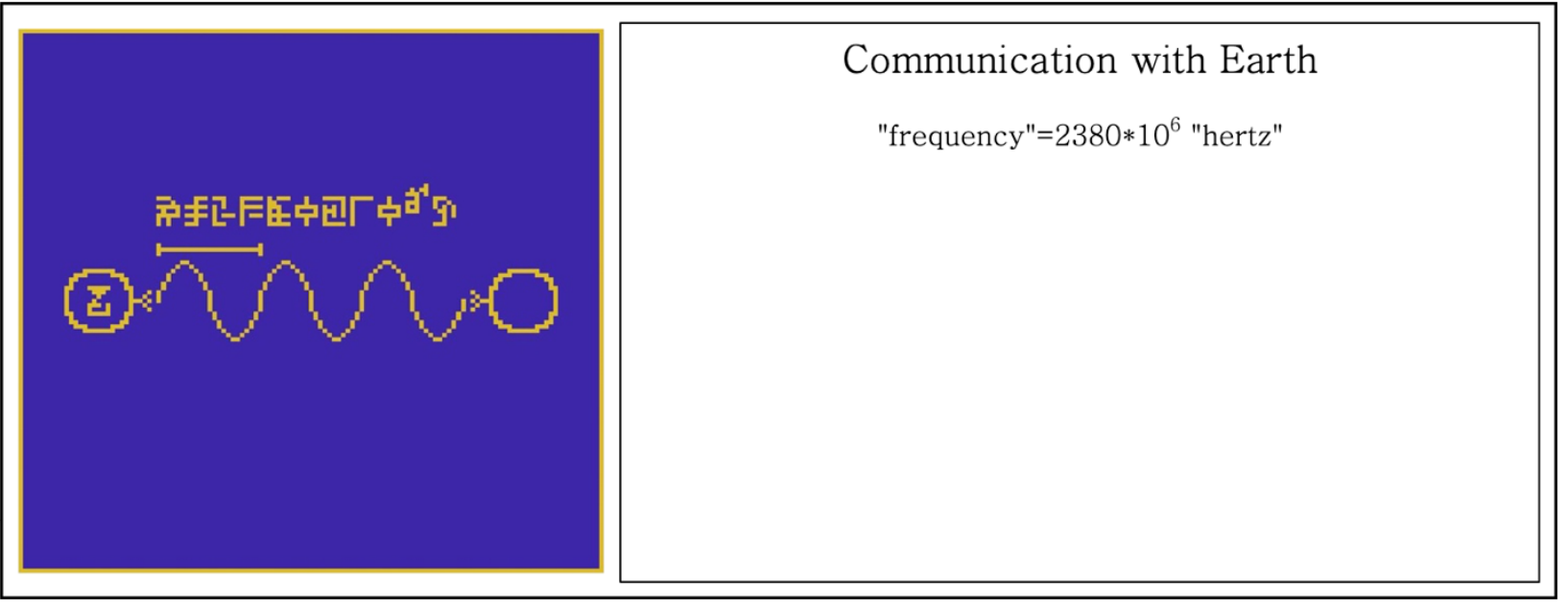

Figure 3.12: Invitation to return a reply in the Message, Page 12. 
Near the end of the message, we convey our location, the point of origin of this message being indicated by globular cluster coordinates. A section of this set of coordinates is shown in Figure 3.13. We know from our own (still very limited) knowledge of exo-planetary systems that one means of characterizing and thus helping to identify host stars can be found in the basic details of the planets which orbit those stars such as mass and semi-major axis. While the BITG message's Solar System depiction is not to scale, logical inferences can be made by noting the number of planets and their relative size. Further, this portion of the message can as well serve as a kind of invitation, complete with our galactic address.

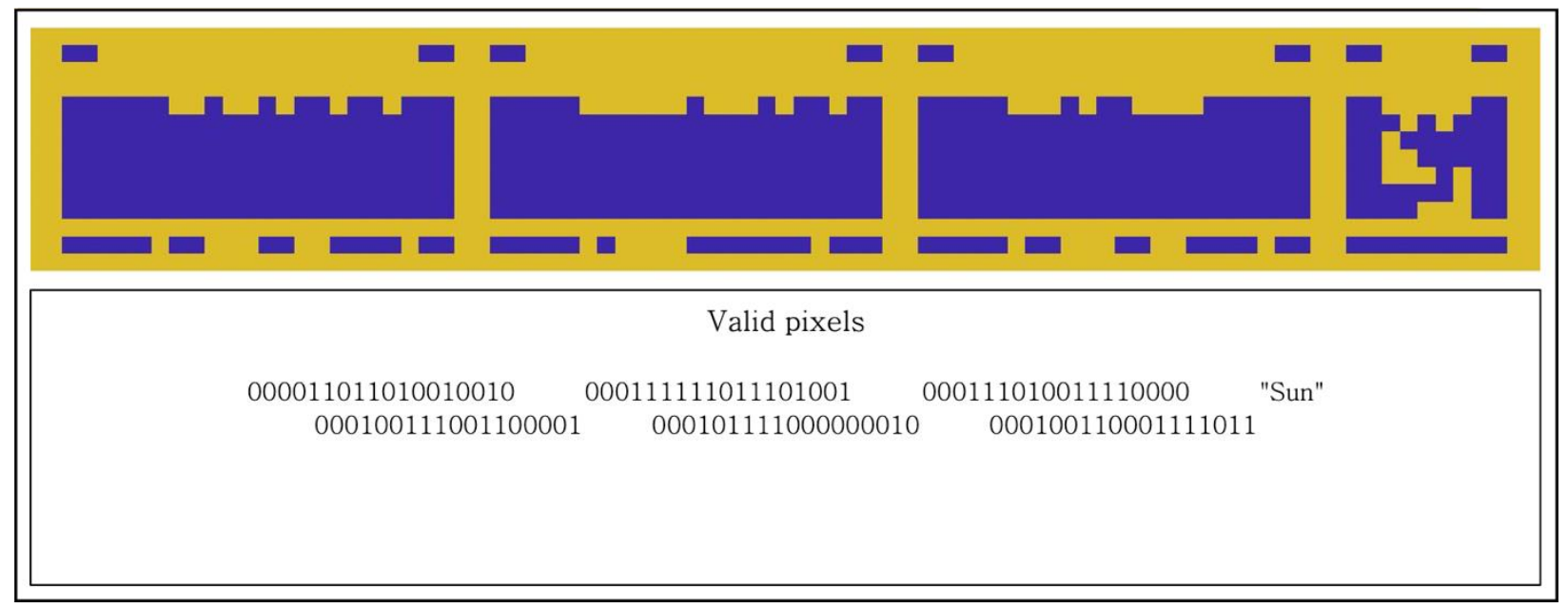

Figure 3.13: A section of the message indicating the position of our Solar system using globular clusters coordinates in the Message, page 13.

\section{Summary}

The Beacon in The Galaxy Message is a scientifically well-crafted communication based off previous works such as the Arecibo Message and Evpatoria Transmissions. The BITG message balances the need for compelling content to pique the interest of the receiver and a scientific basis necessary for understanding the message to make a suitable introduction of humans and, hopefully, enticing a response from any ETI receivers. Unlike the Arecibo Message, which was intended primarily as a technological demonstration of what modern radio astronomy makes possible, the BITG message literally aims for the most likely area in the Milky Way containing intelligent life. By choosing a star cluster between $2 \mathrm{kpc}$ and $6 \mathrm{kpc}$ from the center of the galaxy as the intended destination, we maximize the chances of the message being received by an ETI; thus, we maximize the probability of receiving a response in the distant future.

The message is simple but meaningful. If we were (very hypothetically) to receive a return communication from ETIs, we would expect to see a reciprocation of much of this same information. Perhaps the return message would describe their mathematic system in terms of a different base while still being sent in binary as the BITG message does in explaining our base-10 mathematics. Theoretically, a return message would also include basic information of their own biosphere's chemistry - although it is plausible, they are not carbon based - and an image of their physical appearance. In this scenario, the return message would be a direct analogue to the BITG message and would establish a mutual language to communicate with as well as provide basic, 
essential information for future communications as well as answer many questions of primary interest.

The idea of communication with ETIs is an incredibly intriguing development in the scientific exploration of the cosmos and has only become technically possible in just the last several decades. To seize this opportunity, the BITG message has been ideally designed to be sent by the most advanced of these instruments - the SETI ATA and Tianyan (FAST). Accordingly, its binary coding is concise and maintains the delicate balance needed to further progress of this lofty goal. In the words of Carl Sagan, "Even if the aliens are short, dour, and sexually obsessed-if they're here, I want to know about them." This quote encapsulates the mindset of this message that as scientists we always pursue further knowledge, pushing back the boundaries of the conventional to explore and understand what awaits over the next horizon.

\section{Looking into the Future}

The BITG message is designed with basic information generally outside of change arising from technological and scientific advancements by humans. Hence, the message is a strong candidate to be considered for transmission in the present day even if future studies result in more likely locations of intelligent life in the Milky Way or when humans develop still greater advanced technology capable of sending more powerful transmissions. Such future scenarios may include a network of large radio dishes built in into well-chosen craters on the far side of the Moon, shielded from the cacophony of radio interference from Earth, or highly advanced deep space transmitters capable of sending gravity lensed messages from the radial focal lines which begin 550 AU from the Sun. These future advancements in technology would all greatly boost the transmission power and reduce the effect of radio noise or atmospheric disturbances, allowing for less error in the sent messages as well as the possibility for more distant targets to be chosen. Alternatively, if more compact ways of coding or transmission can be reliably developed (e.g., phase modulation), the message can be edited to fit these criteria while preserving its well-designed contents.

As discussed earlier, this message's ultimate goal is to start a dialogue with ETI - no matter how far in the future that might occur. As this message is meant as an introduction to humans and sent towards what we believe to be an optimal region of the Milky Way for life to flourish, other teams wishing to compose a message of their own can send such to that same region with more complex information. For example, with the basis of communication established, at the next ideal time at which to send a message we would be able to transmit content that includes cultural aspects such as the rules of chess and a mock game to the same location. Alternatively, a future message could include additional frequencies allowing for greater complexity and thus inclusion of some great works of music such the symphonies of Beethoven, Mozart or Bach. Future messages can also include more in-depth discussions of our discoveries in scientific fields; for example, they could include Maxwell's and Einstein's equations or, perhaps, Hawking's blackhole radiation theory or the ideas of dark matter and energy and the expansion of the Universe. In addition to the new information any future messages would convey, it is equally as important to include updated timestamps in each so as to quantitatively distinguish timeframe differences between prior and new messages. These ideas, among others, should be considered as viable follow-ups to the BITG message in the coming years to decades. Humanity has, we contend, a compelling story to share and the desire to know of others' - and now the means to do so. 
Acknowledgement: This work was supported by the Jet Propulsion Laboratory, California Institute of Technology, under contract with NASA. We acknowledge NASA ROSES Exoplanet Research Program for support. We also thanks many supports from the FAST and SETI programs.

Data availability: All data and software used for this study are submitted online as attachments. For additional questions regarding the data sharing, please contact the corresponding author at Jonathan.H.Jiang@jpl.nasa.gov.

\section{References}

[1] - The Staff at The National Astronomy. "The Arecibo Message of November 1974." Icarus, vol. 26, no. 4, 1975, pp. 462-466., doi:10.1016/0019-1035(75)90116-5.

[2] - LALONDE, L. 1 . (1974). The upgraded Arecibo Observatory. c/ence 186, 213-218.

[3] - SAGAS, C. (1975). Communication with Extraterrestrial Intelligence (G. Sagan, Ed.). MIT Press, Cambridge, Mass.

[4] - Kohlhase, C.e., and P.a. Penzo. "Voyager Mission Description." Space Science Reviews, vol. 21, no. 2, 1977, doi:10.1007/bf00200846.

[5] - NASA. "The Golden Record." https://voyager.jpl.nasa.gov/golden-record/

[6] - Sagan, C.; Sagan, L. S.; Drake, F. (1972). "A Message from Earth". Science. 175 (4024): 881-884.

[7] - Dumas, Stephane, and Yvan Dutil. The 1999 and 2003 Messages Explained, The 1999 and 2003 messages explained.

[8] - RAA 2020 Vol. 20 No. 5, 64(28pp) doi:10.1088/1674-4527/20/5/64.

[9] - Harris, William E. "A catalog of parameters for globular clusters in the Milky Way." The Astronomical Journal 112 (1996): 1487.

[10] - Manchester, R. N开, et al. "The Australia telescope national facility pulsar catalogue." The Astronomical Journal 129.4 (2005): 1993.

[11] - Fixsen, D. J. "The temperature of the cosmic microwave background." The Astrophysical Journal 707.2 (2009): 916.

[12] - Riess, Adam G., et al. "Milky Way Cepheid standards for measuring cosmic distances and application to Gaia DR2: implications for the Hubble constant." The Astrophysical Journal 861.2 (2018): 126. 


\section{Appendix A: Globular Cluster Coordinates}

\begin{tabular}{|c|c|c|c|c|c|c|c|}
\hline Coordinate_1 & Separator & Coordinate_2 & Separator & Coordinate_3 & Separator & Note & Line ending \\
\hline 000011011010010010 & Separator & 000111111011101001 & Separator & 000111010011110000 & Separator & Sun symbol & Line ending \\
\hline 000100111001100001 & Separator & 000101111000000010 & Separator & 000100110001111011 & & & Line ending \\
\hline 000011010101111110 & Separator & 000111111101111111 & Separator & 000000001110010010 & & & Line ending \\
\hline 000101111000110001 & Separator & 000011111001010110 & Separator & 000010010111001110 & & & Line ending \\
\hline 000000000001000011 & Separator & 000000110011111101 & Separator & 000001110001110011 & & & Line ending \\
\hline 000000000000000000 & Separator & 000000011001110100 & Separator & 000100111100000000 & & & Line ending \\
\hline 000100111111100110 & Separator & 000000100110101101 & Separator & 000101110100010011 & & & Line ending \\
\hline 000101101110001001 & Separator & 000010010010100011 & Separator & 000101001101010001 & & & Line ending \\
\hline 000011111001010101 & Separator & 000100000110110001 & Separator & 000111111001010000 & & & Line ending \\
\hline 000101110000001100 & Separator & 000100000010000100 & Separator & 000110100001000001 & & & Line ending \\
\hline 000110101100000010 & Separator & 000010001010110011 & Separator & 001100001000100110 & & & Line ending \\
\hline 000110010010100101 & Separator & 000011100110010010 & Separator & 000110100100110110 & & & Line ending \\
\hline 000101111011101110 & Separator & 000100110101000000 & Separator & 001000011000001011 & & & Line ending \\
\hline 000100100101101010 & Separator & 001001000000000001 & Separator & 001111010001011010 & & & Line ending \\
\hline 001001011111011110 & Separator & 000001000101011111 & Separator & 001001000001000101 & & & Line ending \\
\hline 001011101000000010 & Separator & 000101011010010111 & Separator & 001100010101000010 & & & Line ending \\
\hline 000111011111010011 & Separator & 001000001101001111 & Separator & 001011101010010011 & & & Line ending \\
\hline 001000100000101010 & Separator & 000100100100011101 & Separator & 000111110100111111 & & & Line ending \\
\hline 001010100001000000 & Separator & 000011011010111000 & Separator & 000111111011001110 & & & Line ending \\
\hline 010000001100010001 & Separator & 000000000100101010 & Separator & 001000011100100100 & & & Line ending \\
\hline 001010110101001000 & Separator & 000100110010010011 & Separator & 001001001101010111 & & & Line ending \\
\hline 001000110110011010 & Separator & 000100101000100010 & Separator & 000110111111110110 & & & Line ending \\
\hline 001010110110110001 & Separator & 000110111110011000 & Separator & 001001111101100001 & & & Line ending \\
\hline 000101000100101101 & Separator & 000111101010101110 & Separator & 000111110010100110 & & & Line ending \\
\hline 001100001001000100 & Separator & 000000000000000000 & Separator & 000011111101110001 & & & Line ending \\
\hline 001010001010100001 & Separator & 000110111110010110 & Separator & 001001001110011101 & & & Line ending \\
\hline 001011000001001000 & Separator & 000101100000111000 & Separator & 001000010001111011 & & & Line ending \\
\hline 001001101000110100 & Separator & 000110010001011011 & Separator & 001000010110011101 & & & Line ending \\
\hline 001000000101111010 & Separator & 001000001101010011 & Separator & 001001010011001110 & & & Line ending \\
\hline 001001110011101100 & Separator & 000111000001011101 & Separator & 001000101100011000 & & & Line ending \\
\hline 000101100111000011 & Separator & 001011100110000000 & Separator & 001011000000101011 & & & Line ending \\
\hline 000110101101010100 & Separator & 001000110111000001 & Separator & 001001000000001010 & & & Line ending \\
\hline 001010101100110010 & Separator & 000101001010010100 & Separator & 000111000011100110 & & & Line ending \\
\hline 001100010011111010 & Separator & 000111110000111000 & Separator & 001001011100101110 & & & Line ending \\
\hline 000110100001011000 & Separator & 001000110001100000 & Separator & 001000101011100111 & & & Line ending \\
\hline 001011011010011000 & Separator & 000110001100110110 & Separator & 000111110010000001 & & & Line ending \\
\hline 001000101111110010 & Separator & 000111010101001011 & Separator & 000111111111111000 & & & Line ending \\
\hline 001010010100000000 & Separator & 000111100011011110 & Separator & 001000011100110110 & & & Line ending \\
\hline 001111011001110101 & Separator & 000111100101011110 & Separator & 001001011010010100 & & & Line ending \\
\hline 001010110000011001 & Separator & 000111111100101101 & Separator & 001000101111010101 & & & Line ending \\
\hline 001010111001011001 & Separator & 000111100111101110 & Separator & 001000010101101110 & & & Line ending \\
\hline 001000000100110010 & Separator & 000111100101110110 & Separator & 000111101111111010 & & & Line ending \\
\hline
\end{tabular}




\begin{tabular}{|c|c|c|c|c|c|}
\hline 001011101000111000 & Separator & 000111100001101010 & Separator & 001000001000111110 & Line ending \\
\hline 000101011010011000 & Separator & 001100111110001010 & Separator & 001011000101011110 & Line ending \\
\hline 001001100011110010 & Separator & 001000000010010100 & Separator & 001000001011000101 & Line ending \\
\hline 001001100011111101 & Separator & 001000100001110110 & Separator & 001000011110100010 & Line ending \\
\hline 001010000011100110 & Separator & 001000100000000110 & Separator & 001000011100111010 & Line ending \\
\hline 001111001010010000 & Separator & 001001010100010010 & Separator & 001001011100010000 & Line ending \\
\hline 001010101100111011 & Separator & 000111111000010000 & Separator & 001000000000000101 & Line ending \\
\hline 000111100110101000 & Separator & 000110100001011100 & Separator & 000110110000001001 & Line ending \\
\hline 001001010111010101 & Separator & 000111100011001000 & Separator & 000111100011000100 & Line ending \\
\hline 000101111100111010 & Separator & 001000110001110001 & Separator & 001000000101000000 & Line ending \\
\hline 001001001000001010 & Separator & 000111100010001101 & Separator & 000111011100000111 & Line ending \\
\hline 001001111011011001 & Separator & 000111101000111000 & Separator & 000111100011001010 & Line ending \\
\hline 001000001010101110 & Separator & 000100101010110100 & Separator & 000101011110110110 & Line ending \\
\hline 001001111010011001 & Separator & 000111010110000100 & Separator & 000111010010100101 & Line ending \\
\hline 001011111100100011 & Separator & 000110011101000110 & Separator & 000110110010100111 & Line ending \\
\hline 001000101111010011 & Separator & 000111101101001010 & Separator & 000111011001101011 & Line ending \\
\hline 001001110101111110 & Separator & 000110111000111011 & Separator & 000110111010110011 & Line ending \\
\hline 001010111111011001 & Separator & 000101111110101100 & Separator & 000110011000100100 & Line ending \\
\hline 001010000101000011 & Separator & 001010100010001111 & Separator & 001001001100110101 & Line ending \\
\hline 001011110100000110 & Separator & 001000011100000100 & Separator & 000111111001001111 & Line ending \\
\hline 000101000100101101 & Separator & 000111010000111111 & Separator & 000110111011011101 & Line ending \\
\hline 001000000001011110 & Separator & 001000000110011011 & Separator & 000111011100111011 & Line ending \\
\hline 010001010011011000 & Separator & 001111010110000111 & Separator & 001011111001001011 & Line ending \\
\hline 001110010010100000 & Separator & 000111010011001100 & Separator & 000110110101100001 & Line ending \\
\hline 001000111000111010 & Separator & 001000010011001011 & Separator & 000111011110000110 & Line ending \\
\hline 001010000110100000 & Separator & 001000110101110000 & Separator & 000111110000011101 & Line ending \\
\hline 001100100011010101 & Separator & 000110111001010100 & Separator & 000110100000001011 & Line ending \\
\hline 001000110100011010 & Separator & 000111110011000000 & Separator & 000111000110101011 & Line ending \\
\hline 001100100110010101 & Separator & 000111001111100110 & Separator & 000110101011110101 & Line ending \\
\hline 001001100110000101 & Separator & 001000011111001001 & Separator & 000111011001000011 & Line ending \\
\hline 001100000100111100 & Separator & 000110000110000001 & Separator & 000101101111101000 & Line ending \\
\hline 001001000011000111 & Separator & 001000010010010111 & Separator & 000111000111001100 & Line ending \\
\hline 001000011010100110 & Separator & 001000001011000110 & Separator & 000111000101101100 & Line ending \\
\hline 001011010100101101 & Separator & 001010101100001111 & Separator & 001000010011010110 & Line ending \\
\hline 001000000000101110 & Separator & 001000010010101111 & Separator & 000111001001011110 & Line ending \\
\hline 001001100001101000 & Separator & 001000000010101000 & Separator & 000110111000111001 & Line ending \\
\hline 001000001001011010 & Separator & 001010010111010001 & Separator & 001000010010100000 & Line ending \\
\hline 001001101011101010 & Separator & 001000000011100111 & Separator & 000110110110011100 & Line ending \\
\hline 001001001011001110 & Separator & 001010000111110000 & Separator & 001000000010101001 & Line ending \\
\hline 000111100111011100 & Separator & 001000001011000101 & Separator & 000111000100001001 & Line ending \\
\hline 000101110010001111 & Separator & 001000001011001011 & Separator & 000111001101110111 & Line ending \\
\hline 001001001010101000 & Separator & 000110110101111100 & Separator & 000110001001100110 & Line ending \\
\hline 000110001110101010 & Separator & 001000011100111110 & Separator & 000111010100000100 & Line ending \\
\hline 001000001010010010 & Separator & 001000010111100101 & Separator & 000111000011100101 & Line ending \\
\hline 000111010000010011 & Separator & 001000100110000011 & Separator & 000111010001000100 & Line ending \\
\hline
\end{tabular}




\begin{tabular}{|c|c|c|c|c|c|}
\hline 001001010001010000 & Separator & 000111111100111100 & Separator & 000110101100001100 & Line ending \\
\hline 000111011000011100 & Separator & 001001100001011101 & Separator & 000111101110111011 & Line ending \\
\hline 000111001100000110 & Separator & 001000011111001010 & Separator & 000111001010110010 & Line ending \\
\hline 001100000001111000 & Separator & 001000000000010001 & Separator & 000110010011001000 & Line ending \\
\hline 001000100011100000 & Separator & 001000001110111110 & Separator & 000110110101010101 & Line ending \\
\hline 000111101010000010 & Separator & 001001000000000010 & Separator & 000111010000100110 & Line ending \\
\hline 001101001110010101 & Separator & 000100110001010100 & Separator & 000100010001011000 & Line ending \\
\hline 001001101000100000 & Separator & 001000001111000010 & Separator & 000110011100010101 & Line ending \\
\hline 000111101110100111 & Separator & 001000100001011111 & Separator & 000110111000011111 & Line ending \\
\hline 001010110001000000 & Separator & 001000111100111011 & Separator & 000110011000000111 & Line ending \\
\hline 001010010011010010 & Separator & 001000001000111010 & Separator & 000110000011110100 & Line ending \\
\hline 001001101110011001 & Separator & 001001000001100011 & Separator & 000110100101011101 & Line ending \\
\hline 001011001101100111 & Separator & 001000001001000000 & Separator & 000101101111001111 & Line ending \\
\hline 000101111001100000 & Separator & 001000010111011010 & Separator & 000110111110010100 & Line ending \\
\hline 001101001110010111 & Separator & 001010011001011101 & Separator & 000110000110100011 & Line ending \\
\hline 001010011001011010 & Separator & 001000010001111011 & Separator & 000101110000011010 & Line ending \\
\hline 000110010001001000 & Separator & 001001101010110100 & Separator & 000111010011010111 & Line ending \\
\hline 001000010111000101 & Separator & 001010010001110000 & Separator & 000110111001010001 & Line ending \\
\hline 001000110100100101 & Separator & 001001001010110011 & Separator & 000110001111010101 & Line ending \\
\hline 001010000001100001 & Separator & 000111111100001001 & Separator & 000101001111111011 & Line ending \\
\hline 001000011110110111 & Separator & 001011101001001101 & Separator & 000111000100010001 & Line ending \\
\hline 000110000010110010 & Separator & 000110110010010110 & Separator & 000101111011100110 & Line ending \\
\hline 001000001010001100 & Separator & 001011011001010100 & Separator & 000110111001111100 & Line ending \\
\hline 000110110011111011 & Separator & 001110100000100110 & Separator & 001000011001001100 & Line ending \\
\hline 000110010001010110 & Separator & 001011101001101010 & Separator & 000111100010000010 & Line ending \\
\hline 000111010100000100 & Separator & 001000100010010001 & Separator & 000101100111000010 & Line ending \\
\hline 001100001001001110 & Separator & 001101010110010001 & Separator & 000100011100011010 & Line ending \\
\hline 000101001001101100 & Separator & 001010100101100010 & Separator & 000111000011100010 & Line ending \\
\hline 010001011101111000 & Separator & 001101001000011110 & Separator & 000000000101001001 & Line ending \\
\hline 001010101000001110 & Separator & 010001001101000110 & Separator & 000011010010010111 & Line ending \\
\hline 001100101110010011 & Separator & 001110011111100000 & Separator & 000000000000000000 & Line ending \\
\hline 000110100001001011 & Separator & 001110100110011010 & Separator & 000011100000100110 & Line ending \\
\hline 000111110101111100 & Separator & 001101111001001011 & Separator & 000001111101001111 & Line ending \\
\hline 000111010101011100 & Separator & 001001111100100110 & Separator & 000010100110101101 & Line ending \\
\hline
\end{tabular}




\section{Appendix B: The Message}

Page 1-4
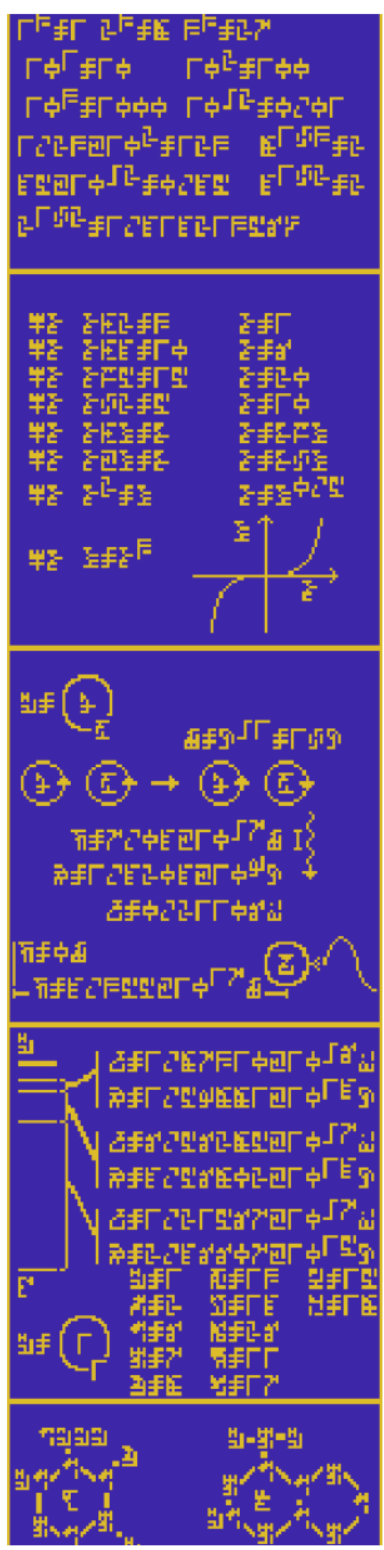

Page 5-8
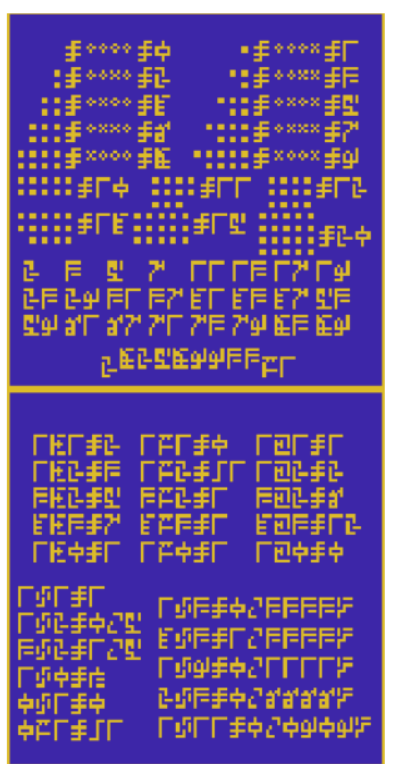

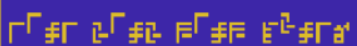

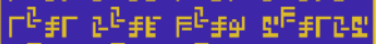

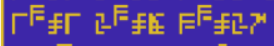

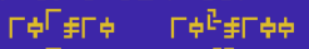

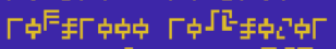

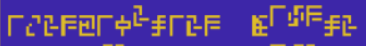

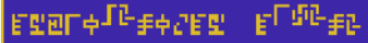

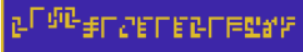

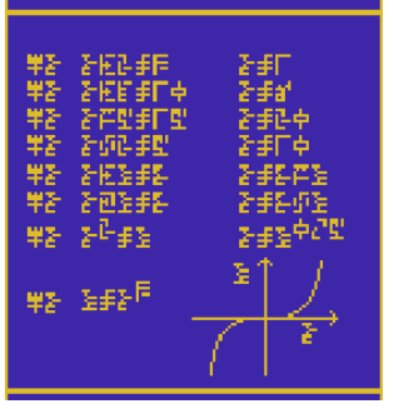

Page 9-13

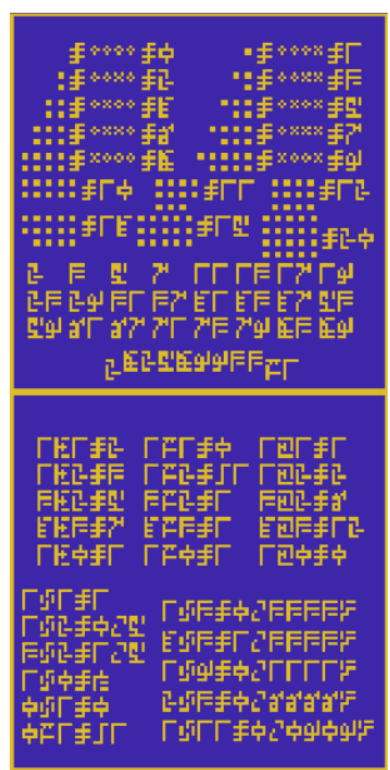

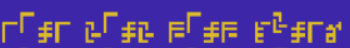

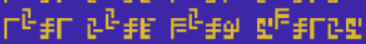

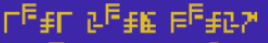

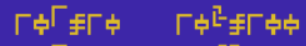

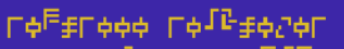

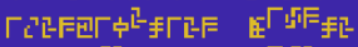

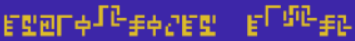

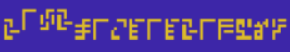
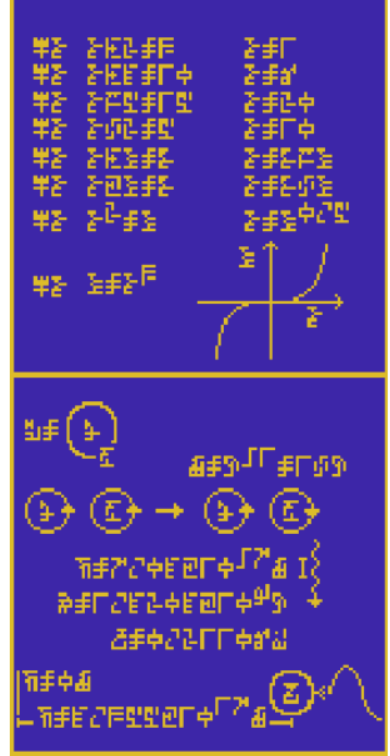

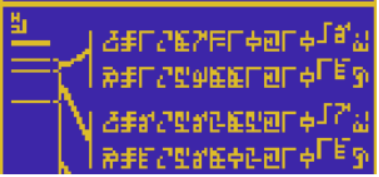

